\title{
6 La fine del mondo nel sesto libro
}

\subsection{Introduzione: il ritorno della tematica escatologica}

Nell'articolazione tematica del De rerum natura, lo spazio riservato alla trattazione sistematica della tematica della mortalità del mondo corrisponde ai vv.91415 del quinto libro. Pertanto, nel proemio del sesto libro (6.43-46) Lucrezio stesso dimostra di ritenere la questione enucleata sotto ogni profilo e quindi apparentemente conclusa:

\footnotetext{
Et quoniam docui mundi mortalia templa esse $\langle$ et $\rangle$ nativo consistere corpore caelum, et quaecumque in eo fiunt fierique necessest, pleraque dissolvi, quae restant percipe porro. Goebel

Poiché ho dimostrato che gli spazi del mondo sono mortali

e ho già in gran parte spiegato che il cielo consiste d'un corpo

soggetto alla nascita e tutto ciò che in esso si compie

e deve accadere, ascolta ora il rimanente.
}

44 et add. 13145 fierique] fateare Lachmann : possuntque Bernays 46 dissolvi] ressolvi

Com'è noto, il sesto libro del De rerum natura è infatti dedicato alla descrizione fondata sul metodo delle spiegazioni multiple ${ }^{1}$ - di alcuni rilevanti fenomeni naturali, capaci d'indurre l'umanità, ignara delle loro reali cause, a cedere ai terrori della religio. ${ }^{2}$ Questa istanza risale a Epicuro stesso, che individua proprio nella mancata comprensione dei fenomeni naturali la radice dello "scivola-

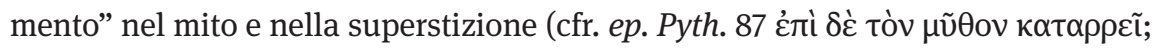
ma anche ep. Hdt. 78) da parte del genere umano. Soltanto l'apprendimento delle reali cause di questi fenomeni può infatti porre l'uomo al riparo dall'ansia, così da raggiungere la beatitudine. ${ }^{3}$ Eppure è proprio nel corso della rappresentazione e della spiegazione dei fenomeni naturali che la tematica escatologica ricompare nel sesto libro, e non si tratta di un'apparizione fugace. In particolare, durante la trattazione dei fenomeni più violenti e terrificanti, ovverosia

1 Cfr. Hardie 2009b, Hankinson 2013 Verde 2013c, Masi 2014 e Bakker 2016 8-75 per un'introduzione alla natura di tale metodo e alla questione della fedeltà di Lucrezio a Epicuro nella ripresa di esso. La relazione tra fine del mondo e spiegazioni multiple verrà indagata più avanti in questo capitolo (cfr. pp. 232-237).

2 Cfr. Lucr. 6.48-55. Per un'introduzione alla meteorologia epicurea cfr. Taub 2009.

3 Cfr. Verde 2013c, 127-130.

Ә OpenAccess. () 2019 Manuel Galzerano, published by De Gruyter. (cc) BY-NC-ND This work is licensed under the Creative Commons Attribution-NonCommercial-NoDerivatives 4.0 License. 
il tuono (vv. 96-159), il lampo (vv. 160-218), il fulmine (vv. 219-422), i terremoti (vv. 535-607) e i vulcani (vv. 639-702), Lucrezio costruisce sistematicamente scenari nei quali il mondo sembra sempre sul punto di crollare, densi di allusioni a miti escatologici (innanzi tutto "gigantomachici”); tale procedimento è accompagnato dalla ripresa - svolta con rinnovata vis polemica - della critica alla teoria dell'eternità cosmica. Questa situazione fa sì che la prima metà del sesto libro - sino alla trattazione del vulcanesimo - sia pervasa dalla tematica della fine del mondo, sebbene in apparenza quest'ultima sia stata già esplicata nel libro precedente.

\subsection{Amplificatio e nec mirum: due opposte tensioni}

Pare opportuno indicare sin da ora che nel costante richiamo a miti e a scenari apocalittici nella prima parte del sesto libro si coglie la compresenza di due opposte tensioni, ovverosia la coesistenza di strategie di rappresentazione in apparenza contraddittorie. ${ }^{4} \mathrm{Da}$ un lato Lucrezio descrive i fenomeni naturali

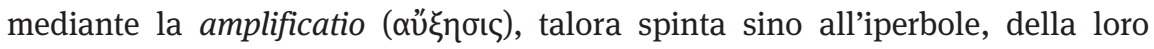
portata distruttiva; in questo modo, il poeta riafferma la fragilità del mondo, al quale, per approdare alla ruina finale, sarebbe sufficiente una catastrofe d'inusitata violenza (la tristior causa già immaginata in 5.346-347). Al contempo, come si vedrà, un altro scopo di tale strategia è anche quello di rivelare i meccanismi psicologici che portano alla genesi del terrore religioso nel cuore di un

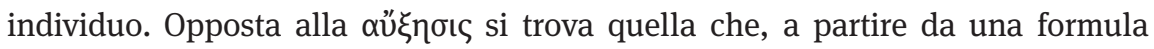
ricorrente nel testo, potremmo definire "strategia del nec mirum"; mediante quest'ultima, l'aspetto spaventoso e misterioso (mirus, appunto) di un fenomeno viene spiegato razionalmente e quindi deprivato della propria eccezionalità, spesso tramite la comparazione "analogica" del fenomeno stesso a situazioni più umili, appartenenti alla realtà quotidiana. ${ }^{5}$

4 Fondamentali in proposito a queste due strategie e alla loro dialettica («the dialectic between these two strategies - the creation and suppression of miratio») le considerazioni di Gale 2000, 198-201: «the DRN is insistent in urging the need to suppress miratio (wonder) through the attainment of ratio. But, at the same time, the poet frequently conveys a strong sense of awe and admiration before the majesty of the natural world [...]. A similar technique is used pervasively in Book 6, where the poet repeatedly creates and deflates a sense of wonder. Phrases which evoke the impressive and even frightening nature of the phenomena under discussion virtually alternate with the formulaic nec or haud mirum».

5 Il richiamo analogico a fenomeni più immediati per spiegare un evento meteorologico che altrimenti sarebbe un åsฤ $\lambda$ ov è connesso alla tecnica epicurea delle spiegazioni multiple, volta, 
Quando Lucrezio ricorre all'amplificatio, il fenomeno naturale viene descritto nelle sue devastanti conseguenze, con un lessico altisonante che guarda innanzi tutto allo stile dell'epica e della tragedia latine. Chiaro è il debito del poeta nei confronti di autori come Ennio, Accio e Pacuvio, ${ }^{6}$ la cui tendenza a mettere in luce la cornice cosmica entro la quale si dipana il dramma dell'umana esistenza viene condotta alle estreme conseguenze. La ripresa di questi modelli di poesia sublime non è gratuita, ma piegata alle profonde ragioni del docere lucreziano. Difatti, è fondamentale notare che questa modalità di rappresentazione viene solitamente costruita a partire da un punto di vista soggettivo. Siamo insomma dinnanzi alla prospettiva di un soggetto che non comprende le ragioni della furia della natura e che pertanto è colto da terrore, precipitando negli incubi della religio o, come direbbe Epicuro, “cadendo nel mito". L'uomo non illuminato dalla dottrina di Epicuro sospetta dunque l'esistenza di una divinità onnipotente e forse ostile all'uomo (cfr. 5.1233 vis abdita quaedam); a tale timore si congiunge il terrore che il mondo, scosso dalle sue fondamenta, pervenga al momento cruciale della propria distruzione. Sebbene tali timori siano irrazionali, dovuti all'ignoranza delle leggi di natura, in essi si cela tuttavia l'intuizione della fragilità del mondo, che così spesso minaccia il crollo. È proprio questa oscura intuizione, non supportata dalla cognizione della verità, a gettare l'uomo nella disperazione e nel terrore. Come si vedrà, l'utilizzo di questa strategia di amplificatio viene diretta anche contro la teoria dell'eternità del mondo, rappresentandol'impotenza di coloro che si ostinano a sostenere tale teoria dinnanzi alla furia degli elementi e all'imminente rovina.

Al contrario, la "strategia del nec mirum" prevede un procedimento di «deflazione del sublime», ${ }^{7}$ ottenuto tramite meccanismi analogici che comparano il fenomeno naturale a un evento quotidiano. Essa viene sempre sviluppata a partire da una "prospettiva cosmica», ${ }^{8}$ dalla quale è possibile comprendere che non vi è differenza qualitativa tra ciò che si osserva in cielo e ciò che si osserva in terra (cfr. e.g. Lucr. 6.48-54), poiché i fenomeni celesti funzionano secondo le

come si è già accennato, a eliminare l'ignoranza delle cause allo scopo etico di condurre l'uomo alla beatitudine.

6 Si confronti, ad esempio, la sequenza di scenari catastrofici elencati da Lucrezio ai vv. 250294 in relazione al fenomeno della tempesta con i vv. 411-416 $\mathrm{R}^{3}$ del Teucer di Pacuvio: inhorrescit mare, / tenebrae conduplicantur, noctisque et nimbum obcaecat nigror, / flamma inter nubes coruscat, caelum tonitru contremit, / grando mixta imbri largifico subita praecipitans cadit, / undique omnes venti erumpunt, saevi exsistunt turbines / fervit aestu pelagus.

7 Riprendo l'espressione da Gale 2000, 198-201.

8 Cfr. Williams 2012, che utilizza la formula «cosmic viewpoint» in riferimento alle Naturales quaestiones di Seneca. Cfr. Andreoni 1978 a proposito del tema della contemplazione nel De re publica ciceroniano. 
medesime leggi fisiche che regolano il mondo terreno. ${ }^{9}$ In questa prospettiva Lucrezio non esita a riutilizzare il modello analogico del $\mu \alpha \kappa \rho \alpha ́ v \theta \rho \omega \pi о$, mettendo in rilievo come il mondo non funzioni diversamente dal corpo umano, anche per quanto concerne i fenomeni più violenti: così, ad esempio, la fenomenologia del terremoto viene comparata ai tremori che colpiscono l'uomo quando prova freddo (cfr. 6.594-595). Tale strategia permette insomma di privare i fenomeni naturali del loro carattere straordinario e quindi d'eliminare quell'horror che è il fondamento del sentimento religioso. Al contempo il lettore, avendo compreso la corrispondenza tra fenomeni celesti e fenomeni terreni, può accettare il fatto che, come i mortali, così anche il mondo nel suo insieme è destinato alla rovina, senza imputarla ad agenti divini. Egli può così giungere a quell'imperturbabilità che è imprescindibile per condurre un'esistenza felice.

Quanto detto dimostra che tali tensioni non risultano affatto antinomiche, bensì complementari. Non pare un caso che la combinazione di queste due strategie trovi la propria espressione più evidente e compiuta proprio durante la trattazione del fenomeno naturale catastrofico del vulcanesimo, indagato nella sezione centrale del libro (vv. 639-702). Qui infatti il poeta prima rappresenta un'eruzione dell'Etna, osservata dalla prospettiva soggettiva delle popolazioni circostanti, che temono un evento distruttivo di portata globale (cfr. v. 644-646) e cedono al terrore irrazionale dinanzi a un dio ostile, volto ad annientare l'umanità (cfr v. 646). Poi però il poeta attua la "strategia del nec mirum" e permette al lettore di vedere il fenomeno dalla giusta angolatura, ovverosia dall'alto (cfr. vv. 647-648): l'eruzione di un vulcano non è diversa da una febbre

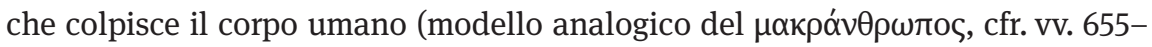
669). Se anche tale malattia conducesse il mondo alla rovina, questa situazione risulterebbe comunque risibile, poiché il mondo è meno di un punto dinnanzi all'infinità dell'universo, che nel suo insieme resta inalterata per l'eternità (cfr. v. 679). Siamo così giunti, almeno momentaneamente, a quella "prospettiva cosmica” necessaria al conseguimento della felicità morale. Il lettore può ora finalmente comprendere ed accettare la natura mortale del mondo, non più esposto ai miti né tanto meno al terrore dinnanzi alla catastrofe. La trattazione dei fenomeni sismici e l'analisi del vulcanesimo costituiscono pertanto il punto di

9 Inevitabilmente, il richiamo tematico alla quotidianità implica un abbassamento del registro lessicale, con un repentino "salto" a un livello linguistico e lessicale umile: come si è già accennato, questo aspetto è tipico del sublime lucreziano, che oscilla costantemente tra diversi livelli. Si vedrà, ad esempio, nell'analisi del fenomeno del tuono, che esso è prima descritto con lessico altisonante chiaramente attinto a modelli epici e tragici, e poi sottoposto a una straniante "deflazione" tramite il paragone con il suono dell'esplosione di una vensicula parva (vv. 130131). 
arrivo ideale del percorso escatologico del lettore lucreziano e il preludio all'ultimo scenario apocalittico del poema, ossia la narrazione della Peste di Atene.

\subsection{Fonti e obbiettivi polemici dei passi escatologici nel sesto libro}

Per quanto concerne le fonti della rassegna lucreziana di $\mu \varepsilon t \varepsilon ́ \omega \rho \alpha$ nel sesto libro, è necessario distinguere i fenomeni naturali trattati nella prima metà (6.96-607) da quelli esaminati nella seconda (vv. 608-1286). La prima presenta infatti notevoli corrispondenze con ben precise fonti epicuree e peripatetiche (o con fonti dossografiche di ascendenza epicurea e peripatetica), mentre la seconda è, come sottolinea Bakker, dedicata a «exceptional and local phenomena,

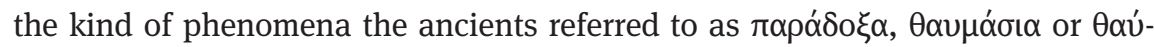
$\mu \alpha \tau \alpha$, and mirabilia or miracula, i.e. 'paradoxes', 'marvels' or 'miracles'»; ${ }^{10}$ per questa ragione, Bakker stesso ha persuasivamente proposto che quest'ampia sezione attinga invece a più recenti fonti paradossografiche, sia che esse siano mediate da un altro autore epicureo, ${ }^{11}$ sia che tale aggiunta costituisca un'originale innovazione lucreziana. ${ }^{12}$

Poiché tuttavia è nella prima metà del sesto libro che si concentrano i più espliciti riferimenti alla querelle sull'eternità del mondo, è dalla questione delle sue fonti, dibattuta sin dal principio del secolo scorso, che bisogna prendere le mosse. Per quanto riguarda le fonti epicuree, il primo termine di riferimento è l'Epistola a Pitocle: essa però, data la sua natura di compendio, rende solo parzialmente conto della rassegna lucreziana e delle sue ragioni. Pare pertanto assai probabile che il modello principale di Lucrezio sia ancora una volta il trattato Sulla natura di Epicuro, i cui frammenti a noi pervenuti a proposito di queste tematiche sono tuttavia rarissimi. ${ }^{13}$ Oltre ai testi epicurei citati, fonda-

10 Cfr. Bakker 2016, 110.

11 Cfr. Bakker 2016, 126.

12 Come sintetizza Bakker 2016, 157, essa può dunque essere ritenuta «a personal innovation by Lucretius in answer to the increasing popularity of such marvel stories in the paradoxographical as well as meteorological literature of his time». Verde 2017b mette in rilievo la persuasività di questa tesi, pur notando che la possibilità di una fonte epicurea intermedia non possa essere esclusa, poiché anche «in Diogene di Enoanda si trova la menzione di luoghi specifici come la Libia, la Scizia, l'Asia e l'India subito dopo la trattazione dei meteora» (cfr. Diog. Oen. NF 182 III (Theol. XVI) Hammerstaedt-Smith).

13 Cfr. Notaro 2006, 8, e Bakker 2016, 93-94 che, riprendendo Sedley, ipotizza che la trattazione

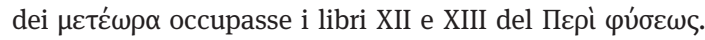


mentali termini di paragone per comprendere questa sezione della meteorologia lucreziana sono i Meteorologica di Aristotele (libri I-III), il trattato pseudo-aristotelico De mundo, i Placita di Aezio (III libro e IV 1), la Naturalis historia di Plinio il Vecchio (2.89-248), la sezione meteorologica stoica di Diogene Laerzio (7.151-154), la Meteorologia siriaco-araba. Bakker ha però dimostrato che soltanto Aezio e la Meteorologia siriaco-araba presentano corrispondenze decisive con i testi di Epicuro e di Lucrezio.

Assai rilevante è dunque la questione dell'attribuzione delle Meteorologia siriaco-araba. ${ }^{14}$ Se infatti tale testo fosse, come considerato dalla communis

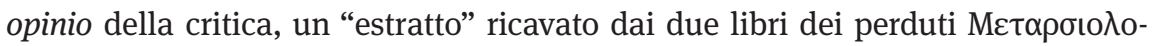
yıкó di Teofrasto, sarebbe inevitabile pervenire alla conclusione di una forte dipendenza di Epicuro e, tramite quest'ultimo, di Lucrezio al tracciato fornito da Teofrasto. ${ }^{15}$ Tale aderenza al modello peripatetico risulterebbe particolarmente significativa per quanto concerne la genesi del metodo delle spiegazioni multiple, presente capillarmente nella Meteorologia. ${ }^{16}$ Sviluppando un'ipotesi di Bergsträsser, Boll e Reitzenstein, ${ }^{17}$ però, Bakker ha rigettato tale interpretazione, proponendo che siano invece i Meteorologica a dipendere da Epicuro. ${ }^{18}$ Il principale argomento in favore di questa tesi consisterebbe nel fatto che nelle re-

14 Per quanto concerne la storia di questo testo, cfr. Verde 2017b: «quest’opera è giunta in versione siriaca e in duplice versione araba rispettivamente di Bar Bahlūl e, forse, di Ibn AlKhammār. Solo nelle due versioni arabe compare il nome di Teofrasto, dato che la corrispondente sezione del testo siriaco è andata perduta. H. Daiber [= D.] ha fornito l'edizione critica delle tre versioni, aggiungendo anche la traduzione inglese della versione araba probabilmente attribuibile a Ibn Al-Khammār». Cfr. dunque anche Daiber 1992, 166-293.

15 Cfr. Sedley 1998a, 179: «since the first partial discovery and publication of this text in 1918 and the subsequent work of Reitzenstein, it has been recognised that it bears a direct causal relation to Epicurean meteorology, especially as we meet this in book 6 of Lucretius».

16 La conclusione inevitabile sarebbe dunque quella sintetizzata da Verde 2017b: «il capillare uso delle molteplici spiegazioni e l'ordine degli argomenti trattati farebbe pensare (come è stato fatto) che Epicuro e Lucrezio dipendano pedissequamente da Teofrasto tanto per l'invenzione del metodo epistemologico delle molteplici spiegazioni quanto per l'ordine dei meteora investigati e la tipologia delle spiegazioni offerte». Come testimonianza di tale lettura si veda Podolak 2010, 39-80.

17 Cfr. Bakker 2016, 71.

18 Cfr. Bakker 2015, 153: «it would seem, then, that we are left with two possible scenario's: either the Syriac meteorology is, as the communis opinio would have it, a version of Theophrastus' lost Metarsiology (allowance made for a certain amount of shortening, omissions, transpositions and perhaps also additions), or it is a compendium largely, but not exclusively, dependent on Epicurean meteorology, into which certain peripatetic elements have been incorporated. How such a compendium came to be transmitted under the name of Theophrastus I could not say, but there is certainly nothing exceptional about such false ascriptions». 
stanti opere di Teofrasto non sia riscontrabile un uso così pervasivo delle spiegazioni multiple. ${ }^{19}$ La conclusione di Bakker è dunque che la più estesa trattazione epicurea dei fenomeni meteorologici nel trattato Sulla natura (dallo studioso definita «Epicurus' other meteorology») rappresenti la fonte della Lettera a Pitocle, di Lucrezio e della Meteorologia siriaco-araba. ${ }^{20}$ Le opere di Teofrasto

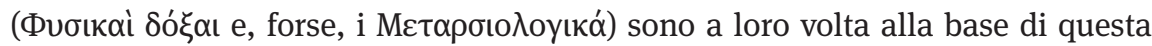
più ampia trattazione meteorologica di Epicuro (come anche di Aezio, Plac. III). ${ }^{21}$ Bakker suppone inoltre che Lucrezio non riprenda solo il modello offerto da Epicuro, ma anche materiale dossografico come quello che si trova in Aezio.

Come si vedrà, pur non giungendo a risposte definitive, l'analisi delle polemiche riferite all'eternità cosmica all'interno di questa sezione può apportare un utile contributo alla definizione degli obbiettivi polemici di Lucrezio nella prima metà del sesto libro.

\subsection{L'immaginario escatologico relativo ai fenomeni del tuono, del lampo e del fulmine}

Si è detto sopra che nel sesto libro la "strategia del nec mirum" convive con l'opposta tendenza ad accrescere la portata "apocalittica” di ogni fenomeno naturale descritto, al punto che ciascuno di essi possa divenire il preambolo del crollo del cosmo. Questa feconda dialettica figura sin dalla trattazione dei primi fenomeni analizzati da Lucrezio: tuono, lampo e fulmine. ${ }^{22}$ Prendendo le mosse dal primo, notiamo che, illustrando gli effetti del tuono, Lucrezio dipinge uno scenario di sconvolgimento dello spazio celeste, che sembra preludere al crollo del cielo e del mondo (cfr. 6.96 quatiuntur caerula caeli; 122-123 divulsa repente/ maxima dissiluisse capacis moenia mundi); non è un caso che l'immaginario e il lessico scelti dal poeta siano i medesimi riscontrati nei passi escatologici dei libri

19 Cfr. Bakker 2016, 70; 146-147. Si noti che Verde 2017b condivide le conclusioni tratte da Bakker: «io credo che, sulla base dei testi, questa ipotesi, per quanto non la si possa provare con certezza, sia particolarmente convincente».

20 Cfr. Bakker 2016, 144-154.

21 Cfr. Bakker 2016, 156-158. Questa tesi presenta tuttavia alcune criticità, dovute in primis al fatto che non tutto il testo della Meteorologia siriaco-araba può essere ricondotto a una prospettiva epicurea, bensì peripatetica. Il caso più notevole è la sezione conclusiva del cap. 14, in cui l'autore del testo si premura di sottolineare che i disordini del nostro mondo non possono essere attribuiti alla divinità (descritta come causa dell'ordine cosmico), bensì alla natura imperfetta di questa parte del cosmo. Cfr. Bakker 2016, 148-153.

22 Per quanto riguarda le fonti di questa sezione, cfr. Notaro 2006, 5-39. Cfr. Bakker 2016, 141143 per un confronto con l'ordine e i contenuti della Meteorologia siriaco-araba. 
precedenti: si notino infatti il richiamo alla repentinità del fenomeno e il ritorno dell'immagine del crollo dei moenia mundi. Questi versi solenni sono però seguiti dall'applicazione della "strategia del nec mirum": alla descrizione di un tuono capace di sventrare la volta celeste segue infatti il riferimento analogico all'umile vensicula parva che esplode (vv. 121-129). ${ }^{23}$ Tale violento salto di prospettiva dimostra l'intento di ridimensionare il carattere straordinario dell'evento.

Non si può però interpretare l'uso in questi versi di lessico e immaginario “apocalittici” come una gratuita e retorica amplificatio nella descrizione di un fenomeno naturale, mossa soltanto da un generico intento parodistico nei confronti dei generi alti del canone letterario; come infatti accennato in sede iniziale, Lucrezio utilizza il suo ricco repertorio di situazioni escatologiche come uno strumento per mostrare la genesi del sentimento religioso nell'animo umano. Siamo insomma dinanzi alla descrizione di un fenomeno naturale a partire da una prospettiva soggettiva - e pertanto parziale - che sopravvaluta la portata del fenomeno stesso. Grazie a questo espediente, Lucrezio permette al proprio lettore di "vedere" e "sentire" gli eventi naturali con gli occhi di quell'umanità descritta nel primo elogio di Epicuro come terrorizzata dalla mostruosa religio e non ancora "redenta": la prima arma della superstizione è infatti la paura dell'uomo, dovuta all'ignoranza, dinanzi alla violenza della natura. Non è un caso che Epicuro venga innanzi tutto elogiato come colui che non ebbe timore dei fulmini e dei tuoni, prima considerati le minacciose armi delle divinità (cfr. 1.68-69 quem neque fama deum nec fulmina nec minitanti / murmure compressit caelum).

Il carattere soggettivo di queste visioni catastrofiche è messo in rilievo dalla presenza ricorrente di espressioni e verbi che veicolano il concetto d'impressione, innanzi tutto videri. Ecco gli esempi più significativi:

- vv. 121-123 tonitru concussa videntur / omnia saepe gravi tremere et divulsa repente / maxima dissiluisse capacis moenia mundi. ${ }^{24}$

23 In riferimento a questo passo cfr. Gale 2000, 199-201.

24 "Anche in tal modo l'intero creato, scosso da un fragoroso tuono, / sembra spesso tremare e l'enorme cerchia di mura / del vasto mondo all'improvviso squarciarsi e crollare». A conferma del chiaro (ed esibito) debito lucreziano in queste sezioni nei confronti della tragedia latina, si vedano i vv. 223-225 Ribbeck dell'Atreus di Accio: sed quid tonitru turbida torvo / concussa repente aequora caeli / sensimus sonere?. Il poeta epicureo riprende dal modello non solo l'espressione tonitru concussa, ma anche il dato cruciale della natura improvvisa e repentina (repente) del fenomeno. 
- vv. 251-252 undique uti tenebras omnis Acherunta reamur / liquisse et magnas caeli complesse cavernas. ${ }^{25}$

- vv. 285-286 quem gravis insequitur sonitus, displosa repente / opprimere ut caeli videantur templa superne. ${ }^{26}$

- Vv. 291-292 omnis uti videantur in imbrem vertier aether / atque ita precipitans ad diluviem revocare. ${ }^{27}$

Lucrezio aveva già anticipato l'uso del medesimo schema nel finale del quinto libro, in particolare nella disamina dello sviluppo della religio nella società umana. Dopo aver infatti individuato nel terrore dinanzi alle manifestazioni naturali più violente la radice della religione, il poeta aveva prospettato un quadro catastrofico (la tragedia dell'induperator), ${ }^{28}$ culminante nel famoso epifonema volto a riassumere l'impressione soggettiva della presenza di una natura ostile: usque adeo res humanas vis abdita quaedam / obterit et pulchros fascis saevasque securis / proculcare ac ludibrio sibi habere videtur (vv. 1233-1235). Anche in questo passo, la scelta del verbo videri in posizione conclusiva dimostra l'intento di Lucrezio di separare nettamente la sfera della soggettività dalla dimensione dell'oggettività. Come sottolineato da Andreoni, una lettura critica ignara di questo punto essenziale ricadrebbe nell'antico pregiudizio interpretativo dell'esistenza di un pessimismo lucreziano. ${ }^{29}$

Tornando all'amplificatio dei fenomeni del tuono, del lampo e del fulmine nel sesto libro, si deve inoltre evidenziare che le allusioni escatologiche sopra delineate sono, nella maggior parte dei casi, dense di richiami (espliciti o meno) all'immaginario escatologico tradizionale, e dunque ai principali miti apocalittici della tradizione letteraria greca e latina. I casi più rilevanti sono i seguenti: - ai vv. 249-252 vi è il richiamo al topos (che sarà caro alla tragedia senecana) della scomparsa della luce e dell'avvento sulla terra delle "tenebre dell'Acheronte", foriere del caos. Come si è giù visto, la confusione tra mondo terreno e mondo dei morti è uno dei motivi topici connessi al ritorno del caos nei miti di Teomachia. ${ }^{30}$

25 «Così da indurci a credere che tutte le tenebre / abbiano lasciato l'Acheronte e riempito le grandi caverne del cielo».

26 «La segue fragoroso tuono, così che sembra / schiantarsi la volta del cielo e crollare dall'alto».

27 «In tal modo l'etere sembra mutarsi in acqua / e così precipitando sommergere in diluvio la terra».

28 Cfr. Appendice 3 e Galzerano 2019b.

29 Cfr. Andreoni 2008, 95.

30 Cfr. e.g. Hom. Il. 20.63-65. 
- ai vv. 253-255 Lucrezio connette la visione in cielo di nubi che paiono "volti di nero terrore" (atrae formidinis ora) e che minacciano di velare il sole (cfr. più avanti v. 265 exempto sole) al mito dei Giganti: come evidenziato dal poeta al principio della sezione escatologica del quinto libro, l'intento di questo ultimi è proprio quello di sovvertire il cielo e di spegnere il sole.

- ai vv. 290-292 si descrivono piogge così intense da richiamare il mito del diluvio universale (cfr. v. 292 ad diluviem revocare).

- ai vv. 374-378 il conflitto tra il calore e il freddo in una tempesta sembra assumere una dimensione cosmica. La discordia e il bellum anceps tra i due elementi riportano infatti il lettore al racconto del conflitto tra acqua e fuoco, diluvio e incendio, svolto nel quinto libro (vv. 380-415). Non si dimentichi che anche la Teomachia omerica nell'Iliade era letta allegoricamente come una rappresentazione del conflitto tra gli elementi naturali.

I casi appena elencati non costituiscono, come si credette nel secolo scorso, l'espressione di un sotterraneo tormento nell'animo del poeta, inconciliabile con la sua formale adesione al luminoso e positivo "credo" epicureo. ${ }^{31} \mathrm{Al}$ contempo, sarebbe errato, penso, cadere nell'opposta tesi interpretativa, considerando questi passi come slegati dal contenuto filosofico e dottrinale del poema, trasformandoli così in compiaciuti sfoggi di allusiva doctrina e di abilità retorica, tesi a stupire i lettori. Le allusioni ai miti escatologici, sempre connesse alla fase dell'amplificatio dei fenomeni naturali, sembrano invece impiegate a uno scopo ben preciso: quello d'illustrare che l'esito inevitabile di una visione dei fenomeni naturali non "illuminata" dalla ratio epicurea è la ricaduta nel mito paventata da Epicuro in ep. Pyth. 87. Lucrezio sembra anzi suggerire (quasi "palefatianamente”) la chiave per comprendere la genesi di molti conturbanti miti escatologici della tradizione: esemplare il caso degli atrae formidinis ora (vv. 253-255), che mostrano come certi scenari di tempesta abbiano potuto evocare, nelle menti degli uomini, inquietanti sogni di assalti al cielo da parte di esseri mostruosi. Quanto detto non deve condurre alla conclusione che Lucrezio escluda anche una fruizione estetica di questi passi; semplicemente, il sublime escatologico lucreziano non è mai fine a se stesso, ma “incanalato" a un intento etico, e rappresenta, come si vedrà, una tappa (non la fine) del viaggio conoscitivo intrapreso dal lettore del De rerum natura. ${ }^{32}$

31 A proposito del successo di tale schema interpretativo (anche in relazione a questi versi), cfr. Rumpf 2003, 19-28.

32 Lo scopo della costante oscillazione del lettore lucreziano tra una prospettiva soggettiva e una prospettiva cosmica (nella quale, a mio parere, risiede la quintessenza del "sublime" lucreziano, cfr. pp. 252-270) è infatti di natura didattica e morale: essa mira cioè a rendere il lettore 
Come già accennato nella premessa di questo capitolo, l'abbondanza d'immagini escatologiche in questa sezione ubbidisce a un altro scopo, ovverosia mettere in rilievo la fragilità del mondo, le cui possibili cause di distruzione sono molteplici. Tale obbiettivo è inevitabilmente connesso con la polemica filosofica implicita in questi passi, diretta contro la dottrina peripatetica dell'eternità del cosmo. Infatti, come nei passaggi escatologici dei libri precedenti, la trattazione della tesi epicurea è sempre mossa da una vis demistificatrice nei confronti delle teorie a essa opposte. Certo, la dottrina della mortalità del mondo è stata già dimostrata nel libro precedente, ma la rassegna di fenomeni meteorologici violenti nella prima metà del sesto libro consente al poeta di mostrare che, data la possibilità dell'afflusso nel mondo di qualsiasi tipologia di atomi proveniente dall'immensità esterna, ogni evento naturale può trasformarsi in una tristior causa capace di condurre il mondo alla rovina (cfr. 5.345-347). Nel corso del sesto libro l'enfasi lucreziana sulla portata "apocalittica" di numerosi eventi naturali è dunque volta a ribadire, di fenomeno in fenomeno, questa verità, imprimendola a fuoco nell'animo del lettore.

\subsection{I fenomeni sismici (vv. 535-607)}

$\mathrm{Al}$ v. 535 prende avvio la sezione dedicata alla natura e alle cause del terremoto (vv. 535-607). Si tratta di una sezione fondamentale per comprendere l'escatologia lucreziana, visto che la maggior parte degli scenari apocalittici del poema sembra prevedere l'avvento di un terribile sisma, tale da provocare il crollo dei moenia mundi e il ritrarsi della terra sub pedibus. ${ }^{33}$ Pare innanzi tutto opportuno presentare una possibile suddivisione di questa sezione:

- vv. 535-542. Presentazione dell'argomento.

- vv. 543-551. Alcuni terremoti sono causati da crolli delle caverne sotterranee (addirittura d'intere montagne) dovuti alla consunzione provocata dal

lucreziano capace ed avvezzo a compiere autonomamente il "volo" epicureo oltre i moenia mundi, a trascendere le limitate prospettive terrene.

33 Rappresentazioni di sismi catastrofici si ritrovano infatti in: 1.1105-1107 (neve ruant caeli tonitralia templa superne / terraque se pedibus raptim subducat et omnis / inter permixtas rerum caelique ruinas); 2.1144-1145 (sic igitur magni quoque circum moenia mundi / expugnata dabunt labem putrisque ruinas); 5.104-106 (dabit ipsa fidem res / forsitan et graviter terrarum motibus ortis / omnia conquassari in parvo tempore cernes); 5.340 aut cecidisse urbis magno vexamine mundi; 5.1236-1240 denique sub pedibus tellus cum tota vacillat / concussaeque cadunt urbes dubiaeque minantur, / quid mirum si se temnunt mortalia saecla / atque potestates magnas mirasque relinquunt / in rebus viris diuum, quae cuncta gubernent?. 
tempo. Per spiegare il fenomeno, nei vv. 548-551 il poeta impiega lo schema del nec mirum, richiamando l'exemplum quotidiano del carro che varca una via, facendo tremare le case attorno.

- vv. 552-556. Alcuni terremoti sono causati invece da moti di acque sotterranee, dovuti a frane. I vv. 555-556 contengono l'exemplum del vaso che oscilla, perché colmo d'acqua in movimento.

- vv. 557-576. Un'altra causa dei sismi è il moto violento del vento nelle spelonche sotterranee, che insiste su un punto solo, provocando l'inclinazione della superficie terrestre. Lucrezio descrive poi gli esiti catastrofici di questo tipo di terremoti nelle città. I vv. 565-574 sono una prima parentesi diretta contro gli assertori dell'eternità del mondo: lo scopo del poeta è infatti quello di sottolineare la fragilità del mondo, che è sopravvissuto sino ad oggi solo perché «la terra minaccia rovina più spesso di quanto non abbia ad attuarla» (vv. 572-573 saepius minitatur terra ruinas / quam facit).

- vv. 577-590. Vi sono anche terremoti che sono dovuti al vento che (sorto dall'esterno o dall'interno) tumultua nel sottosuolo e infine squarcia la superficie terrestre, talora inghiottendo intere città, come Egio nel Peloponneso e Sidone in Siria.

- vv. 591-595. Talvolta l'aria che tumultua sotterra non riesce ad aprire voragini, ma si dirama attraverso i fitti forami del suolo, causando un tremore che è paragonato al tremore delle membra umane, quando provano freddo.

- vv. 596-600. Quadro apocalittico finale. La prima parte descrive il terrore degli abitanti delle città dinanzi al terremoto.

- vv. 601-607. Seconda parte del quadro apocalittico finale. Descrizione polemica della resipiscenza degli assertori dell'eternità cosmica, che, dinanzi alla violenza del terremoto, non riescono a trattenere il timore che il mondo sia prossimo alla distruzione.

Dopo questa sintesi pare opportuno volgersi a un esame più approfondito di questa sezione. ${ }^{34}$ Il primo passo degno di nota per la nostra disamina sono indubbiamente i vv. 543-551, che descrivono i terremoti dovuti a crolli sotterranei; qui troviamo alcuni degli elementi ricorrenti nella descrizione lucreziana del terremoto: in primis la repentinità del fenomeno (v. 546 repente); l'articolazione della catastrofe su un piano superiore (v. 544 superne) e un piano inferiore (v. 545 subter); l'enfasi sulle ruinae provocate dal sima (v. 544 magnis ruinis; v. 545 subruit). Come si sarà notato, il lessico e le modalità scelte dal poeta ri-

34 Per un introduzione alle principali fonti di questo passo cfr. Verde 2013c, 137-140, Bakker 2016, 104-105. 
chiamano i numerosi passi escatologici presenti nei libri precedenti; se, ad esempio, paragoniamo questa descrizione al grande cataclisma descritto nel finale del primo libro, ritroviamo una serie di notevoli analogie: anche qui si riscontra infatti l'enfasi sulla natura improvvisa del fenomeno (v. 1103 subito; v. 1109 temporis puncto), l'articolazione alto-basso (vv. 1105-1106 caeli templa superne [...] terra se subducat), il richiamo alle ruinae (v. 1105 neve ruant; v. 1107 inter permixtas ruinas). Tali corrispondenze intertestuali permettono di comprendere come per Lucrezio vi sia una relazione diretta tra ogni sisma e il terremoto finale, che condurrà il mondo alla rovina: tale relazione verrà ribadita al termine di questa sezione. I vv. 543-551 sono inoltre degni di nota, poiché in essi il poeta applica "lo schema del nec mirum": il terrore sovrannaturale provocato dalla catastrofe viene ridimensionato mediante l'analogia con un fenomeno della quotidianità: i vv. 548-551 mostrano infatti come il tremore e il boato del terremoto siano paragonabili agli effetti dei carri che varcano fragorosamente le strade. Non vi è quindi bisogno di ricorrere alla divinità o al mito per spiegare il tremor della terra.

La sequenza successiva (557-595) è così densa d'immagini escatologiche, che pare opportuno considerarla nella sua interezza, senza ripartizioni:

Praeterea ventus cum per loca subcava terrae 557 collectus parte ex una procumbit et urget obnixus magnis speluncas viribus altas, incumbit tellus quo venti prona premit vis 560 Tum supera terram quae sunt exstructa domorum ad caelumque magis quanto sunt edita quaeque, inclinata minent in eandem prodita partem protractaeque trabes impendent ire paratae. Et metuunt magni naturam credere mundi exitiale aliquod tempus clademque manere, cum videant tantam terrarum incumbere molem! Quod nisi respirent venti, vis nulla refrenet res neque ab exitio possit reprehendere euntis. Nunc quia respirant alternis inque gravescunt

Et quasi collecti redeunt ceduntque repulsi, saepius hanc ob rem minitatur terra ruinas quam facit; inclinatur enim retroque recellit et recipit prolapsa suas in pondera sedis. Hac igitur ratione vacillant omnia tecta,

Summa magis mediis, media imis, ima perhilum.

Est haec eiusdem quoque magni causa tremoris, ventus ubi atque animae subito vis maxima quaedam aut extrinsecus aut ipsa tellure coorta in loca se cava terrai coniecit ibique speluncas inter magnas fremit ante tumultu 
versabunda〈que) portatur, post incita cum vis exagitata foras erumpitur et simul altam diffindens terram magnum concinnat hiatum in Syria Sidone quod accidit et fuit Aegi in Peloponneso, quas exitus hic animai disturbat urbis et terrae motus obortus. Multaque praeterea ceciderunt moenia magnis motibus in terris et multae per mare pessum subsedere suis pariter cum civibus urbes.

Quod nisi prorumpit, tamen impetus ipse animai ut fera vis venti per crebra foramina terrae dispertitur ut horror et incutit inde tremorem; frigus uti nostros penitus cum venit in artus, concutit invitos cogens tremere atque movere.

558 urget $O^{1}$ : urges $O Q 563$ minent] manent $Q^{1}$ : tument $A$ : meant Lachmann : minantur eandem in Goebel eandem 131 : eadem $O Q$ post partem a.a. q.q. $O$ : aqueq. $Q$ (? ex quaeque 562) 565 et $Q:$ te $O \quad 568$ venti, vis Marullus : ventis $O Q$ refrenet $A B$ : refremet $O$ : refert met $Q \quad 574$ pondera $Q$ : pondere $O \quad 582$ que add. 131583 exagitata] est agitata Diels 584 diffindens $/ 31$ : diffidens $O Q \quad 586$ quas Avancius : qua $O:$ que $Q \quad 588$ ceciderunt Mon. : cecideret $O$ : caecideret $Q \quad 589$ pessum $Q^{1}:$ possum $O^{1} Q: \operatorname{dossum} O$ 594 uti $Q^{1}:$ ut in $O Q$

Inoltre se il vento, raccolto nelle cavità sotterranee, prorompe e incalza in un'unica direzione, premendo con irresistibile forza sulle profonde spelonche, la terra si piega dove incombe l'impeto del vento. Allora le abitazioni che sono edificate sul suolo, e in maggior misura quelle costruite più alte, pendono recline e minacciano di rovinare verso la medesima parte, e le travi fuoriescono oblique pronte a cadere.

E alcuni si rifiutano di credere che la natura del mondo sia attesa da un tempo di morte e da una rovina finale, pur vedendo incombere una così immensa massa di terra! Se i venti non spirassero a intervalli, nessuna forza potrebbe frenare le cose, né riafferrarle dalla loro corsa al disastro. Ora poiché spirano e si rafforzano con alterna vicenda, e quasi adunati tornatno ad assalire, ma cadono respinti, per tale causa la terra minaccia rovina più spesso di quanto non abbia ad attuarla; infatti s'inclina, ma torna a ritrarsi e riaccoglie nelle sue sedi il peso traboccante. Dunque tutti gli edifici vacillano in questo modo: in alto più che in mezzo, in mezzo più che in basso, in basso quasi per nulla. V'è anche un'altra causa di grandi terremoti, quando il vento o un improvviso possente impeto dell'aria, sorto dall'esterno o dal seno stesso della terra, s'avventa nelle cavità del suolo, e qui dapprima freme in tumulto tra le vaste caverne e imperversa 


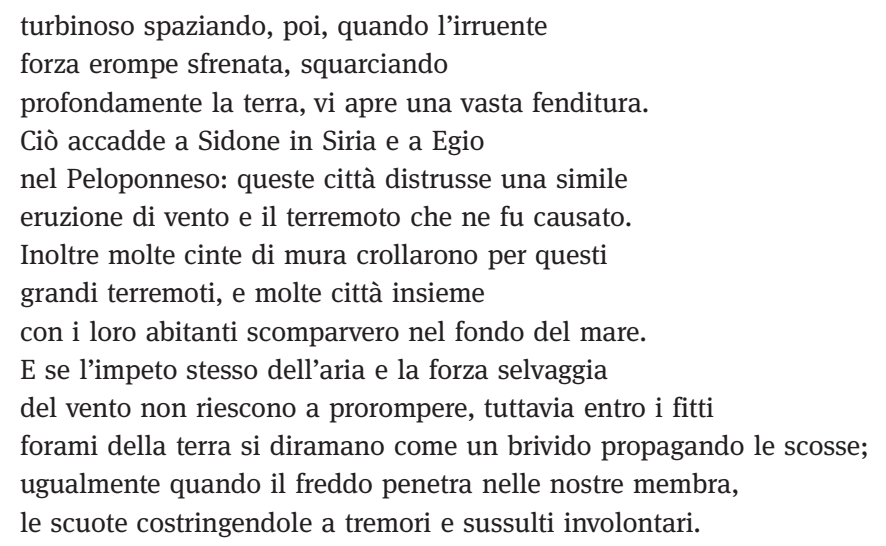

I vv. 557-564 avviano la trattazione relativa alla tipologia di terremoti a cui Lucrezio, sul solco di Epicuro, rivolge più attenzione, ovverosia quelli provocati dai venti sotterranei: nel passo si trova innanzi tutto la distinzione tra loca subcava terrae (v. 557), nonché le profonde caverne in essi celate (v. 559) e gli spazi al di sopra di esse (v. 561 supera terram); stavolta però il poeta concentra subito la propria attenzione sugli effetti catastrofici che il terremoto ha sulle città (vv. 561-564). Dinanzi a tanta ruina il poeta prorompe nell'esclamazione polemica dei vv. 565-567, diretta contro coloro che, pur assistendo a catastrofi di questa portata, non riescono ad ammettere l'idea che il mondo sia mortale e atteso da un exitiale aliquod tempus forse immanente. Il lessico di questo passo rimanda indubbiamente alla trattazione del libro precedente, in particolare ai vv. 338-347, dove il poeta, riportando - come si è visto - una risposta alla difesa teofrastea dell'eternità del mondo, asserisce che è proprio la gravità delle catastrofi che colpiscono il mondo a provare la sua mortalità. Anche in questo passo del quinto libro vi è infatti un riferimento al crollo delle città (v. 340 cecidisse urbis) e si ritrovano per giunta i sostantivi exitium e clades per indicare l'incombente distruzione del mondo (v. 344; 347). Nel medesimo passo del quinto libro Lucrezio mette in rilievo che - accettata la dottrina di periodiche catastrofi cosmiche - è facile intuire come basterebbe una minaccia più grave per condurre tutto alla distruzione. Parimenti, i vv. 566 ss. del sesto libro sottolineano come i terremoti non abbiano ancora disgregato il mondo soltanto perché i venti sotterranei non soffiano costantemente (nisi respirent venti); il fatto che talora i venti esauriscano la propria spinta prima di provocare la catastrofe fa sì che non sempre la minaccia si concretizzi. . $^{35}$

35 Tale principio spiega un passo problematico del quinto libro, ovverosia i vv. 105-107: qui 
Al riferimento ai terremoti di Sidone e di Egio, ormai topici nella trattatistica concernente questo tipo di fenomeni, ${ }^{36}$ segue la riproposizione dell'immagine di città che crollano a partire dalle mura e di città che affondano in mare con i propri abitanti: è assai probabile che qui vi sia un rimando alla catastrofe che travolse le città di Elice e Bura, già descritto da Callistene di Olinto. Avvicinandosi alla conclusione del passaggio, il poeta applica nuovamente lo "schema del nec mirum", volto a ridimensionare il terrore provocato da scenari così sconvolgenti. Stavolta, però, Lucrezio non rappresenta situazioni della vita quotidiana solo quantitativamente distinte dal fenomeno in questione, bensì sceglie di ricorrere all'argomento analogico del $\mu \alpha \kappa \rho \alpha ́ v \theta \rho \omega \pi о \varsigma$, mostrando come il corpo del mondo funziona in modo simile al corpo umano. ${ }^{37} \mathrm{Il}$ tremito della terra durante un terremoto diviene così simile all'incoercibile tremore (horror) che prende il corpo dell'uomo quando è esposto al freddo. Come si è detto, tale similitudine potrebbe essere ripresa anch'essa dalle fonti peripatetiche, come Callistene di Olinto e il De mundo. ${ }^{38}$ A differenza di queste fonti (e forse in polemica con esse), Lucrezio sviluppa questo meccanismo analogico in maniera non neutra, bensì veicolando implicitamente l'idea della mortalità del mondo. Se infatti l'analogia tra certi terremoti e le vibrazioni provocate da un carro (vv. 543-551) aveva un mero scopo illustrativo, in questo caso il nesso tra il

Lucrezio prospetta la possibilità che il grande terremoto che distruggerà il mondo abbia luogo in parvo tempore, sotto gli occhi del suo destinatario. Il poeta si augura però che la fortuna gubernans procrastini il più possibile tale sciagura. Il passo del sesto libro ci permette di comprendere come il riferimento alla fortuna gubernans sottenda un richiamo a quegli eventi fisici casuali, non controllabili da parte dell'uomo (si ricordi la dottrina del clinamen), che determinano la gravità delle catastrofi. Tuttavia la divinizzazione della fortuna, implicita nell'epiteto gubernans, è, come si è visto, intrisa d'ironia nei confronti degli avversari dell'Epicureismo.

36 Cfr. Sen. nat. 6.24.6 e 6.25.4: quid dicam; cum Chalcis tremuit, Thebas stetisse? Cum laboravit Aegium, tam propinquas illi Patras de motu audisse? Illa vasta concussio quae duas suppressit urbes, Helicen et Burin, circa Aegium constitit. Apparet ergo in tantum spatium motum pertendere quantum illa sub terris vacantis loci inanitas pateat.

37 Questo particolare uso dello schema analogico che lega il mondo al corpo umano era già stato compiuto sistematicamente nel finale del secondo libro, dove la crescita del mundus era stata paragonata alla crescita del corpo, nutrito dal cibo. Una significativa somiglianza si riscontra però nuovamente con i vv. 338-350 del quinto libro, che si concludevano appunto con un analogia tra la morte del mondo e la morte dell'individuo, regolate dai medesimi meccanismi (vv. 348-350).

38 L'analogia tra il corpo dell'uomo e il corpo del mondo fu ereditata e sviluppata anche dagli Stoici: in relazione ai fenomeni sismici, si pensi ad esempio all'ampio passaggio del sesto libro delle Naturales quaestiones (6.14.1-4) dove Seneca riporta un'estesa comparazione tra il meccanismo di tali fenomeni e il "funzionamento" del corpo umano quando è colto dalla malattia. 
cosmo e un corpo umano soggetto a malattia è svolto sulla base del denominatore comune della fragilità. Tale analogia comunica indirettamente al lettore che anche il mondo, come un individuo, è esposto alle minacce provenienti dallo spazio esterno e che dunque anch'esso è destinato alla morte. Questa conclusione sarà oggetto di una rappresentazione esplicita nel quadro conclusivo della sezione (v. 596-607):

\footnotetext{
Ancipiti trepidant igitur terrore per urbis, tecta superne timent, metuunt inferne cavernas terrai ne dissolvat natura repente, neu distracta suum late dispandat hiatum atque suis confusa velit complere ruinis. Proinde licet quamvis caelum terramque reantur incorrupta fore aeternae mandata saluti; et tamen interdum praesens vis ipsa pericli subdit et hunc stimulum quadam de parte timoris, ne pedibus raptim tellus subtracta feratur in barathrum rerumque sequatur prodita summa funditus et fiat mundi confusa ruina.
} 600

600 idque] adque Lachmann complere $O^{s}$ : comple $O Q \quad 604$ subdit $O$ : subdita $Q$ et hunc] adhuc Lachmann parte $O^{1}$ : parti $O Q \quad 605$ subtracta $L$ : substructa $O Q$ : fortasse subducta (cf. i.1106)

E dunque la gente nelle città trema per un duplice timore, che i tetti si abbattano dall'alto, e che la natura della terra a un tratto disgreghi le caverne che si aprono nel sottosuolo, e squarciandosi spalanchi in se stessa un vasto abisso, e in tale sconvolgimento voglia colmarlo con le proprie rovine. Quindi pensino pure che al cielo e alla terra incorruttibili sia riservata un'eterna durata; tuttavia la stessa forza presente del pericolo insinua da qualche parte uno stimolo di paura, che la terra mancata all'improvviso sotto i piedi, sprofondi nel baratro e la segua la totalità delle cose sconvolte dalle fondamenta e si produca una confusa rovina del mondo.

Si tratta di un passo di carattere drammatico e non dottrinale, dove il poeta rappresenta un evento catastrofico “apocalittico” e, al contempo, la genesi del terrore nel cuore di coloro che assistono alla catastrofe. Non si dimentichi che siamo all'interno di una conclusione, ovverosia una di quelle posizioni privilegiate dove Lucrezio ama porre sublimi scenari escatologici; come nel finale del secondo libro (vv. 1150-1174 grandis arator e il vitis sator) e nel quadro dell'induperator al termine del quinto (vv. 1226-1235), anche qui al centro dello scenario si trovano personaggi che non comprendono le reali ragioni del fenomeno 
naturale cui assistono e pertanto sono presi da disperazione e terrore. ${ }^{39}$ Eppure nel sesto libro si assiste a una variazione nell'utilizzo di questo schema: nei primi due casi citati Lucrezio attaccava la tradizionale Weltanschauung romana, incarnata dai contadini e dal generale; qui, invece, l'obbiettivo polemico è un altro. Al principio pare che il poeta si riferisca genericamente a coloro che vivono nelle città (v. 596 per urbis), ma poco dopo vediamo che in verità il vero obbiettivo sono gli assertori di un'opinione ben specifica (v. 601-602 caelum terramque reantur / incorrupta fore aeternae mandata saluti). Tale espressione riprende quella analoga del quinto libro (v. 1215) divinitus aeterna donata salute, (riferito ai moenia mundi) e sintetizza la tesi dell'eternità del mondo. Come già aveva intuito Bignone, qui i primi avversari di Lucrezio sono indubbiamente i filosofi peripatetici. Persino la scelta lessicale del poeta rimanda alla tradizione peripatetica; in particolare sembra opportuno richiamare il notevole parallelo offerto da alcune locuzioni presenti nel trattato pseudo-aristotelico De mundo: si

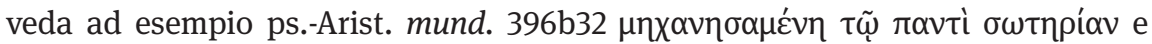

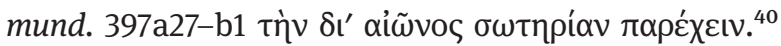

Si noti inoltre che il fatto che il poeta scelga i filosofi come obbiettivo polemico del passo potrebbe essere interpretato come un ulteriore motivo di continuità tra Lucrezio ed Epicuro. Nell'Epistola a Pitocle (85) Epicuro evidenzia infatti che anche coloro che indagano i fenomeni naturali, ma sono ignari delle

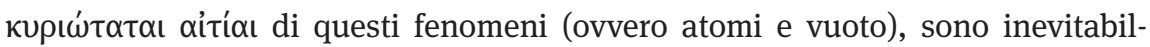
mente travolti da paure e turbamenti; è anzi la loro conoscenza dettagliata, ma

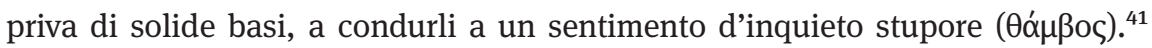

Posti dinanzi alla grande minaccia del terremoto, i filosofi rappresentati da Lucrezio perdono la fede granitica nella teologia astrale. Il sentore del pericolo incombente (v. 603 praesens vis pericli) s'insinua nell'animo di questi uomini, inducendoli a temere la confusa ruina del mondo, mentre la terra si sottrae sotto i piedi e tutto scompare nel baratro. Ciò non significa che il terremoto rappresentato da Lucrezio sia realmente quello che condurrà il mondo alla distruzione:

39 Cfr. Giancotti 1989, 142 e Galzerano 2017. Si tratta dello schema che in Appendice 3 definisco "finimondo con spettatore". Ritroviamo un quadro molto simile in Sen. nat. 6.29.1-2, dove Seneca rappresenta la reazione degli uomini dinanzi alla catastrofe del terremoto. Il filosofo mette in rilievo che, dinanzi alla furia della natura durante il sisma, nessuna persona è al riparo dallo sconvolgimento mentale (nemo sine aliqua iactura sanitatis expavit); sono le menti deboli che però sono destinate alla follia (et in dementiam transfert). Pare notevole che Lucrezio, a differenza di Seneca, dipinga il terremoto come fonte di terrore anche per i filosofi (ovviamente avversari), che in esso intuiscono confusamente la verità della mortalità cosmica.

40 Cfr. Appendice 4. Questo non esclude che il poeta includesse tra i propri obbiettivi polemici anche gli Stoici, che avevano accettato la dottrina della $\sigma \omega \tau \eta \rho i ́ \alpha$ cosmica.

41 Cfr. Verde 2013c, 129. 
si noti che l'intero quadro apocalittico dei vv. 605-607 è introdotto dalla congiunzione ne, che palesa la natura del timore insinuatosi nel cuore dei filosofi. Si tratta invece di un altro caso di "impressione di finimondo", analoga a quelle sopra elencate e accomunate dal ricorrere del verbo videri. Tuttavia a differenza di questi ultimi passaggi, quello dei vv. 601-607 è caratterizzato dall'assenza di una strategia del nec mirum; tale assenza è imputabile a tre cagioni di ordine diverso:

- Una ragione "estetica”: la conclusione della sezione abbisogna di una chiusura “apocalittica” e sublime (v. 607 et fiat mundi confusa ruina), scevra di rimandi a realtà umili e quotidiane.

- Una ragione “polemica”: l'amplificatio del terremoto da parte dei Peripatetici cela l'intuizione della natura mortale del mondo. Questi versi conclusivi si configurano così come definitiva confutazione della dottrina dell'aeterna mundi salus.

- Una ragione “didattica”: come avverrà nel finale del sesto libro, è compito del lettore del poema, ormai giunto al termine di un percorso iniziatico di comprensione del mondo, trascendere autonomamente da una prospettiva soggettiva ad una prospettiva cosmica, ponendosi al riparo dai terrores di questo mondo.

\subsection{I vulcani (vv. 639-702)}

Nunc ratio quae sit, per fauces montis ut Aetnae expirent ignes inter dum turbine tanto, 640 expediam; neque enim mediocri clade coorta flammae tempestas Siculum dominata per agros finitimis ad se convertit gentibus ora, fumida cum caeli scintillare omnia templa cernentes pavida complebant pectora cura, quid moliretur rerum natura novarum. Hisce tibi in rebus latest alteque videndum et longe cunctas in partis dispiciendum, ut reminiscaris summam rerum esse profundam et videas caelum summai totius unum quam sit parvula pars et quam multesima constet nec tota pars, homo terrai quota totius unus. quod bene propositum si plane contueare ac videas plane, mirari multa relinquas. numquis enim nostrum miratur, siquis in artus 655 accepit calido febrim fervore coortam aut alium quemvis morbi per membra dolorem? opturgescit enim subito pes, arripit acer 
saepe dolor dentes, oculos invadit in ipsos, existit sacer ignis et urit corpore serpens

quam cumque arripuit partem repitque per artus, ni mirum quia sunt multarum semina rerum et satis haec tellus morbi caelumque mali fert, unde queat vis immensi procrescere morbi. sic igitur toti caelo terraeque putandumst

ex infinito satis omnia suppeditare, unde repente queat tellus concussa moveri perque mare ac terras rapidus percurrere turbo, ignis abundare Aetnaeus, flammescere caelum; id quoque enim fit et ardescunt caelestia templa et tempestates pluviae graviore coortu sunt, ubi forte ita se tetulerunt semina aquarum. 'at nimis est ingens incendi turbidus ardor.' scilicet et fluvius qui visus maximus ei, qui non ante aliquem maiorem vidit, et ingens arbor homoque videtur et omnia de genere omni maxima quae vidit quisque, haec ingentia fingit, cum tamen omnia cum caelo terraque marique nil sint ad summam summai totius omnem. Nunc tamen illa modis quibus inritata repente flamma foras vastis Aetnae fornacibus efflet, expediam. Primum totius subcava montis est natura fere silicum suffulta cavernis. Omnibus est porro in speluncis ventus et aër. Ventus enim fit, ubi est agitando percitus aër. hic ubi percaluit cale fecitque omnia circum saxa furens, qua contingit, terramque et ab ollis excussit calidum flammis velocibus ignem, tollit se ac rectis ita faucibus eicit alte. Fert itaque ardorem longe longeque favillam 690

differt et crassa volvit caligine fumum extruditque simul mirando pondere saxa; ne dubites quin haec animai turbida sit vis. Praeterea magna ex parti mare montis ad eius radices frangit fluctus aestumque resolvit. Ex hoc usque mari speluncae montis ad altas perveniunt subter fauces. Hac ire fatendumst et penetrare mari penitus res cogit aperto atque efflare foras ideoque extollere flammam saxaque subiectare et arenae tollere nimbos. In summo sunt vertice enim crateres, ut ipsi nominitant, nos quod fauces perhibemus et ora. 
641 mediocri clade Vossius : media grecia de $O Q$ : media de clade Ital. 642 flammea Nonius : flamme $O$ : flamma $Q \quad 648$ dispiciendum $L$ : despiciendum $O Q 653$ propositum $Q^{1}$ : propositus $O Q$ plane $Q^{1}$ : plani $O Q \quad 662$ quia $O^{1}$ : qui $O Q \quad 663$ morbi] nobis Marullus : orbi Lachmann 667 concussa $O^{1}$ : concusso $O Q \quad 674$ qui visus] quivis est Bentley : qui visu est Merrill 676 omni $/ 31$ : omnis $O Q \quad 683$ fere silicum $O^{1}$ : feres illi cum $O Q 684$ et aer $O$ : aether $Q \quad 687$ contingit $l 31$ : contigit $O Q \quad 690$ itaque Heinsius : itque OQ 695 resorbet $l 31$ : resolvet $O Q \quad 697^{\star} 698$ lacunam indicavit Munro 698 mari] animam Van der Valk penitus] ventum Merrill res cogit aperto] res cogit aperta Creech : percocta in apertum Lachmann : alii alia 701 vertice enim Turnebus : verticeni $O$ : vertigeni $Q$

Or qual sia la ragione per cui attraverso le fauci del monte Etna spirino talvolta fuochi così turbinosi, io chiarirò. Esplosa con immoderata strage, la tempesta di fiamme, imperversando pei campi dei Siculi, fece volgere a sé gli sguardi delle genti vicine, quando, vedendo scintillare fumidi tutti gli spazi del cielo, sentirono colmarsi i cuori d'angoscioso terrore, chiedendosi quali spaventosi eventi preparasse la natura. In questa materia si deve essere di larghe e profonde vedute, e scrutar lontano con lo sguardo in tutte le direzioni, per ricordare la nozione che la somma delle cose è infinita, e intendere quanto mai piccola, quale infinitesima parte dell'infinito costituisca quest'unico cielo, neanche tutta la parte che un solo uomo è nei confronti di tutta la terra. Su tieni l'attenzione ben ferma su tale premessa, e discerni con chiarezza, cesserai di stupirti di molti fenomeni. Forse ci meravigliamo infatti, se alcuno di noi accoglie nelle membra una febbre che risorge con bruciante arsura o un'altra qualsiasi dolorosa infermità nel corpo? Si gonfia un piede d'un tratto, un acuto dolore s'insinua nei denti, invade persino gli occhi, spunta il fuoco sacro e serpeggiando nero brucia qualsiasi parte invade, e striscia per le membra; né v'è da stupirsi, poiché esistono semi d'innumerevoli cose, e questa terra e il cielo producono morbi e mali bastanti, da cui possa accrescersi il furore di un'infermità senza limiti. Così dunque devi credere che alla terra e al cielo ogni cosa è provveduta dall'infinito in quantità sufficiente per cui la terra d'improvviso possa squassata tremare, e sulle acque marine e sui campi trascorra un vertiginoso turbine, e la lava dell'Etna trabocchi e il cielo avvampi.

Infatti accade anche ciò, gli spazi celesti fiammeggiano, e le tempeste di pioggia si producono con più grave scroscio, quando così per caso si dispongono i semi dell'acqua. "Ma è eccessivo" dirai "l'ardore turbinoso di questo incendio". Certamente, ma è anche così per un fiume che appaia il più grande 


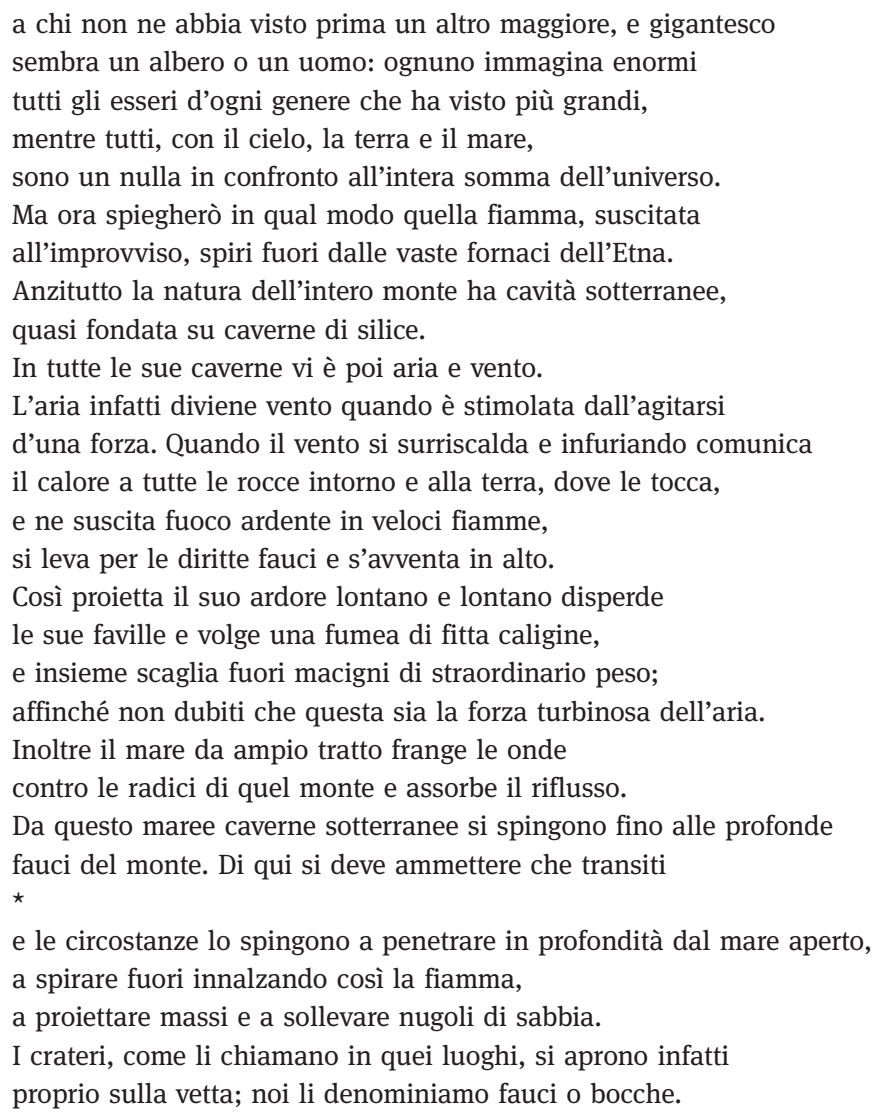

La spiegazione dei fenomeni vulcanici si trova nel punto centrale del sesto libro (la cui entità complessiva è di 1286 versi) e ne costituisce in qualche modo il cuore pulsante: come anticipato nel capitolo precedente, infatti, in questa sezione Lucrezio fornisce la chiave di lettura della rassegna di fenomeni naturali presente nell'intero libro e "rivela" il punto d'arrivo del percorso conoscitivo compiuto dal suo lettore. Come per la sezione precedente, pare opportuno, innanzi tutto, definire una partizione di questi versi fondamentali:

- vv. 639-641. Introduzione.

- vv. 641-646. Quadro “apocalittico” incipitario, che raffigura le popolazioni della Sicilia mentre assistono, da lungi, alla furia di un'eruzione vulcanica dell'Etna, che pare minacciare finanche il cielo.

- vv. 647-654. Al fine di rimuovere lo stupore e il terrore dinanzi alla potenza della natura ("strategia del nec mirum", cfr. v. 654 mirari multa relinquas), 
Lucrezio esorta il lettore a una "prospettiva cosmica”, così da comprendere la nullità della terra rispetto all'infinito universo.

- vv. 655-672. La "strategia del nec mirum" s’imposta ora sullo schema analogico del $\mu \alpha \kappa \rho \alpha ́ v \theta \rho \omega \pi о$ s. Il corpo umano può essere soggetto a innumerevoli malattie, che sopraggiungono dall'infinito spazio circostante. Analogamente, il mondo può essere soggetto a svariate tipologie di fenomeni catastrofici. Lucrezio non si limita al vulcanesimo, ma allarga lo sguardo a buona parte dei fenomeni violenti descritti nel sesto libro: il terremoto (v. 667), i turbini (v. 668), le eruzioni vulcaniche (v. 669), le tempeste e il diluvio (vv. 671-672).

- vv. 673-679. Lucrezio immagina poi l'obiezione di un anonimo interlocutore (v. 673) che non riesce a trovare una ragione adeguata per spiegare l'eccessiva furia del fuoco. La risposta del poeta consiste nel ribadire che, da una prospettiva cosmica, nessun fenomeno naturale appare nimis ingens.

- vv. 680-702. Chiusa l'ampia parentesi avviata al v. 647, Lucrezio si volge alle cause del vulcanesimo, proponendo solamente una spiegazione: nelle caverne sotterranee l'aria tumultua sino a divenire un vento infuocato che spande fiamme dalle rocce e prorompe dai crateri.

L'importanza di questo passo nell'economia generale del libro è confermata dal fatto che la vera e propria trattazione dei fenomeni vulcanici è relegata negli ultimi versi (vv. 680-702), ${ }^{42}$ mentre i primi quaranta versi sono invece dedicati all'enunciazione del passaggio a una prospettiva cosmica, fornendo una chiave di lettura di tutti i fenomeni naturali prospettati nella prima metà di questo libro. ${ }^{43}$ Difatti, sin dal principio (vv. 647-654), il poeta compie un'operazione molto simile a quella svolta nei vv. 1114-1117 del primo libro e nei vv. 1044-1051 del secondo, dove egli aveva parimenti promesso al lettore una libera e luminosa "visione" del cosmo infinito. Pare notevole che tali promesse erano collocate anche in questi casi a ridosso degli scenari escatologici finali, quasi a garantire al lettore la presenza di un porto sicuro dal quale poter osservare il naufragio del mondo. In questi versi del sesto libro la dimensione visiva della rivelazione

42 La struttura di questa sezione è in qualche modo invertita rispetto a quella della sezione sui terremoti. La descrizione di un quadro apocalittico che mette in scena il fenomeno in questione è infatti collocata all'inizio, subito seguita dal richiamo al $\mu \alpha \kappa \rho \alpha ́ v \theta \rho \omega \pi о \varsigma$ e dall'invito a una visione cosmica dell'evento. L'analisi più "scientifica" del fenomeno naturale è stavolta situata in posizione conclusiva (vv. 680-702) e priva d'immaginario apocalittico o di riferimenti alla querelle sull'eternità del mondo. Per questa ragione, essa non sarà oggetto di analisi in questa sede.

43 Per un commento al passo, cfr. Rumpf 2003, 208-222. 
epicurea viene ancor più sottolineata (videndum; dispiciendum; videas; contueare) e presentata come conditio sine qua non della fine della meraviglia (e dunque del timore) dinnanzi ai fenomeni naturali (v. 654 mirari multa relinquas). La scelta dei verbi videre e despicere crea inoltre un nesso intratestuale con il proemio del secondo libro, dov'erano utilizzati per descrivere la visione impassibile del saggio sul mondo (vv. 7-10): sed nil dulcius est, bene quam munita tenere / edita doctrina sapientum templa serena, / despicere unde queas alios passimque videre / errare atque viam palantis quaerere vitae. ${ }^{44}$

Altrettanto rilevanti sono i versi successivi (vv. 655-672) che richiamano per l'ennesima volta l'analogia tra l'essere umano e il mondo; come però avveniva nella sezione sui terremoti e, ancor prima, nel quinto libro, l'uso del $\mu \alpha \kappa \rho \alpha ́ v-$ $\theta \rho \omega \pi$ s non è affatto neutro: il vero scopo dell'autore non è qui quello di spiegare le cause di un fenomeno, bensì quello di far comprendere al suo lettore che il denominatore comune tra l'essere umano e il mondo è, in sostanza, la mortalità. Il notevole elenco delle malattie colpiscono l'uomo (vv. 655-664) trova così un perfetto rispecchiamento nell'elenco di morbi che possono colpire il cosmo (vv. 665-673): l'evidente sottotesto è che tutti questi morbi possono essere causa di morte per il mondo. Si ricordi che nel quinto libro, in polemica contro i Peripatetici, il poeta aveva sottolineato la mortalità del mondo usando come exemplum la natura umana, soggetta a svariate malattie (vv. 348-350). Lucrezio sceglie inoltre come malattie del mondo "per eccellenza" l'incendio e il diluvio, ovverosia gli stessi fenomeni che, sempre nel quinto libro, erano definiti come causa precipua di distruzione del cosmo (vv. 380-415).

Dato che Lucrezio costruisce sempre il proprio argomentare a partire dalla demolizione di una prospettiva erronea, non epicurea, anche in questo caso risulta fondamentale il quesito a proposito dell'identità degli avversari di Lucrezio. Se guardiamo ai primi versi, notiamo che, più che uno specifico avversario, Lucrezio sembra qui attaccare un "sentire comune" ai propri contemporanei, secondo cui lo scatenarsi delle catastrofi naturali è l'esplicazione di un nume invincibile e misterioso, capace di annientare l'umanità. Tale visione è sunteggiata nello splendido quadro incipitario, dove si rappresenta un'eruzione vulcanica dell'Etna attraverso gli occhi atterriti delle popolazioni confinanti che la osservano inquiete (cfr. v. 643). La contemplazione dell'erompere del fuoco

\footnotetext{
44 Il despectus lucreziano, inteso come "sguardo dall'alto" rivolto alla natura, può essere interpretato come perfetto contraltare del concetto di ن் che Damiani 2016, 16-17 definisce come «la paura e la preoccupazione nei confronti dei fattori che destano inquietudine [...]: i meteora, la morte, gli dèi, la minaccia che può pervenire dagli altri uomini». Il percorso ideale del lettore del poema si configura in fondo come una progressivo elevazione dalla ن்тофí al despectus nei confronti dei fenomeni naturali.
} 
sotterraneo, capace addirittura di raggiungere le regioni celesti e minacciare l'ordine cosmico (cfr. v. 644), riempie i loro cuori di angoscia e terrore (cfr. v. 645): essi giungono così ad immaginare l'esistenza di una natura ostile, onnipotente e imperscrutabile (cfr. v. 646 quid moliretur rerum natura novarum). Si noti che il poeta si era già avvalso di una simile personificazione della natura nemica dell'umanità nella sezione escatologica del quinto libro, all'interno della sezione relativa appunto alla culpa naturae (vv. 195-234).

Quanto detto non esclude però che questo passo possa contemplare anche una polemica filosofica. ${ }^{45} \mathrm{Nel}$ corso di quella che abbiamo definito "strategia del

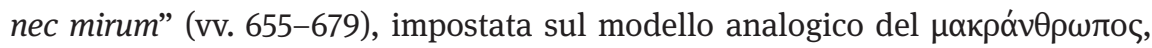
Lucrezio immagina infatti l'obiezione di un anonimo interlocutore, che asserisce che il turbidus ardor dell'incendio dell'eruzione è smodato (v. 673 nimis est ingens). Si tratta di una replica in fondo sciocca ed inconsistente, dato che essa avviene quando il poeta ha appena spiegato che l'universo è infinito e dunque innumerevoli atomi di ogni tipo possono affluire nel mondo. Il ricorso all'occupatio al v. 673 ha dunque lo scopo retorico di corroborare in maniera definitiva il concetto che il mondo in tutta la sua grandezza (si noti l'enfasi sulla sua tradizionale tripartizione al v. 678) è nulla, se comparato all'immensità dell'universo. Tuttavia le parole del v. 673 potrebbero rappresentare anche il punto di vista di qualcuno che non è in grado di concepire il fatto che gli elementi del nostro mondo non siano distribuiti in maniera armonica ed equilibrata, senza alcuna predeterminazione divina. La parola chiave del verso è infatti l'avverbio nimis, che enfatizza l'idea dell'eccesso.

I passi esaminati nei capitoli precedenti inducono a supporre che l'accento lucreziano sull'idea di un disordine cosmico dovuto a un elemento eccedente rispetto agli altri possa essere contrapposta innanzi tutto alla dottrina creazionistica platonica esposta nel Timeo. Una delle condizioni fondamentali dell'immortalità cosmica secondo Platone è infatti proprio la distribuzione armonica ed equilibrata dei quattro elementi nel mondo, grazie al piano provvidenziale del Demiurgo ( $\mathrm{Ti}$. 32c-33d). Tale perfetta isonomia, dovuta anche al fatto che i quattro elementi sono stati utilizzati nella loro intera quantità, fa sì

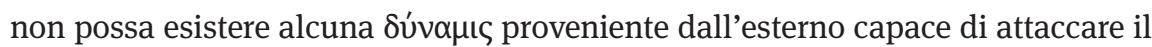
cosmo e arrecare distruzione (cfr. contra Lucr. 666-667 ex infinito satis omnia suppeditare). Credo però che questi argomenti platonici siano attaccati in quanto radice dell'analoga visione peripatetica, che a partire dal medesimo presupposto

45 Per quanto concerne i richiami allusivi al mito di Tifeo in questi versi e il loro legame con l'auctoritas escatologica empedoclea, associata al medesimo mito in $1.722-725$, si veda l'Appendice 2. 
argomentava l'eternità del mondo. Si vedano ad esempio i capp. 21-27 del De aeternitate mundi, dove un anonimo peripatetico giunge a richiamare l'auctoritas dei passi platonici appena citati; ${ }^{46}$ oppure si considerino gli argomenti di Critolao in aet.70-75, che parimenti dimostra che il mondo non può essere soggetto a cause di rovina dall'interno o dall'esterno. Del resto, è verosimile che gli obbiettivi polemici di questa sezione siano i medesimi delle precedenti, ossia coloro che caelum terramque reantur / incorrupta fore aeternae mandata saluti (vv. 601-602). Un'ulteriore conferma del fatto che Lucrezio, nella sua trattazione sui vulcani, si contrapponga a una trattatistica peripatetica viene offerta da un passo del De mundo, nel quale, subito dopo l'analisi dei fenomeni sismici, si riporta un aneddoto a proposito di un'eruzione dell'Etna a riprova del governo provvidenziale del cosmo. ${ }^{47}$

Per quanto possiamo intuire dalla breve obiezione del. v. 673 e dalla conseguente risposta del poeta, gli avversari qui contrastati non sembrano contemplare la nozione di uno spazio infinito esterno rispetto al quale il mondo "è nulla" (vv. 678-679). Ciò induce a supporre che il poeta non stia qui rivolgendo la propria attenzione agli Stoici, che invece riconoscevano l'infinità del tutto. Non si dimentichi inoltre che gli Stoici difendevano la dottrina dell'ecpirosi, che di fatto consisteva nella periodica preponderanza dell'elemento igneo sugli altri elementi; al contrario l'innominato avversario di Lucrezio rifiuta l'idea che l'ardor divenga nimis ingens. ${ }^{48}$

\subsection{Gli avversari di Lucrezio nel sesto libro}

Le considerazioni svolte offrono un quadro abbastanza chiaro a proposito dell'identità degli avversari di Lucrezio nella rassegna di fenomeni meteorologici che occupa la prima metà del sesto libro: si tratta dei difensori della tesi dell'eternità del mondo, ossia Aristotele e i suoi successori. La polemica si fa

46 Cfr. Runia 1986. Anche nel De mundo, dopo una rassegna di fenomeni naturali che include fenomeni catastrofici come il terremoto (cfr. 396a) l'autore riafferma la natura armonica e immortale del cosmo (396b20-397a1) dovuta all'azione della divinità che garantisce l'armonia e

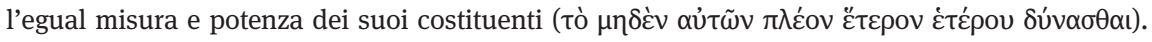
47 Tale aneddoto verrà riportato nell'Appendice 4.

48 Ciò non esclude che gli Stoici possano essere intesi come obbiettivo polemico in senso lato, in quanto assertori della provvidenzialità del cosmo. Come si è visto, le polemiche antiprovvidenzialistiche di Lucrezio tendono a essere più inclusive che esclusive nei confronti degli avversari. 
infatti esplicita in due punti che pare opportuno riportare nuovamente (6.573575; 6.601-607):

Et metuunt magni naturam credere mundi
exitiale aliquod tempus clademque manere,
cum videant tantam terrarum incumbere molem!
[...]
Proinde licet quamvis caelum terramque reantur
incorrupta fore aeternae mandata saluti;
et tamen interdum praesens vis ipsa pericli
subdit et hunc stimulum quadam de parte timoris,
ne pedibus raptim tellus subtracta feratur
in barathrum rerumque sequatur prodita summa
funditus et fiat mundi confusa ruina.

Il pensiero degli avversari di Lucrezio è riassunto nei vv. 601-602 mediante la formula caelum terramque reantur / incorrupta fore aeternae mandata saluti: come si vedrà più in dettaglio nell'Appendice 4, essa sembra costituire la fedele traduzione di analoghe espressioni che si leggono nel trattato pseudo-aristotelico De mundo. Appare dunque chiaro che il poeta sta riprendendo alcune parole d'ordine della cosmologia avversaria allo scopo di sovvertirla radicalmente. Tale procedimento è riscontrabile in entrambi i passi citati: si noti infatti che Lucrezio attribuisce ai propri avversari un timore sotterraneo, quasi inconfessabile (v. 673 metuunt; v. 604 stimulum timoris): quello del crollo del cosmo. ${ }^{49}$ Questo riferimento polemico sarebbe incomprensibile, se non si chiamasse in causa il frammento più noto del perduto De philosophia (fr. 18 Ross), nel quale Aristotele stesso afferma di «essere spaventato da coloro che con il loro ragionamento

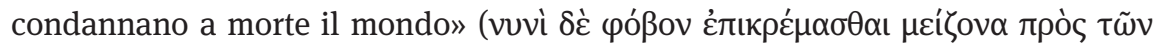

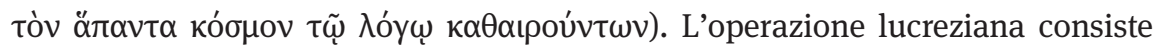
dunque nel "mettere in scena" la progressiva $\mu \varepsilon \tau$ óvoı $\alpha$ di questi pensatori dinnanzi alla vis ipsa pericli, rivelazione dell'assenza di una divinità provvidenziale pronta a salvaguardare il mondo.

L'analisi dei fenomeni meteorologici nella prima metà del sesto libro è dunque sfruttata come un'occasione per ribadire la natura mortale del mondo in opposizione esplicita ad Aristotele, Teofrasto e i loro successori. ${ }^{50}$ Volgendo lo

\footnotetext{
49 A proposito dei motivi del "peso opprimente" e del crollo nel De rerum natura, cfr. Segal 1998, 185-192.

50 Si noti che, come nel secondo e nel quinto libro, anche nel sesto l'attacco alla dottrina aristotelica dell'eternità del cosmo sia accompagnato da un passo volto a negare il provvidenzialismo: si tratta della parentesi dei vv. 379-422. Questi versi, a differenza di quelli dedicati al tema della mortalità del cosmo, trovano un perfetto parallelo nella Meteorologia siriaco-
} 
sguardo ai paralleli epicurei proposti da Bakker (l'Epistola a Pitocle e la Meteorologia siriaco-araba) si nota come invece in questi testi l'analisi dei fenomeni meteorologici non sia mai utilizzata in riferimento alla dottrina della mortalità del cosmo. Come spiegare allora questi passaggi polemici nel poema lucreziano? Due sembrano i quadri più probabili: ${ }^{51}$

- Lucrezio deriva i riferimenti alla dottrina della mortalità cosmica dal trattato Sulla natura di Epicuro; data la loro natura di compendio, tali riferimenti sono invece assenti in testi quali l'Epistola a Pitocle e la Meteorologia siriacoaraba.

- Lucrezio introduce autonomamente il tema della mortalità del mondo all'interno della rassegna meteorologica. L'assenza di riferimenti a tale argomento nell'Epistola a Pitocle e nella Meteorologia siriaco-araba testimonia un analogo disinteresse da parte di Epicuro all'interno della propria rassegna meteorologica nel trattato Sulla natura.

Nel primo caso, Lucrezio deriverebbe le proprie argomentazioni anti-peripatetiche da Epicuro, mentre nel secondo esse sarebbero imputabili a uno sviluppo indipendente da parte del poeta, da ricondurre alla predilezione lucreziana nei confronti della tematica escatologica. La soluzione qui prospettata è analoga a quella fornita a proposito dei vv. 235-380 del quinto libro. ${ }^{52}$ Vi sono infatti buone ragioni per supporre che il nucleo dell'attacco contro l'eternità cosmica in queste

araba, nella seconda parte del capitolo 14: cfr. Mansfeld 1992a, 326-327; Bakker 2016, 151-153. Anche in questo caso, però, è possibile cogliere l'intento lucreziano di attualizzare una polemica teologica risalente a Epicuro: i vv. 380-382, che rappresentano infatti la disperata consultazione dei Tyrrhena carmina, ovverosia le dottrine divinatorie e le profezie etrusche (non Tyrrhena retro volventem carmina frustra / indicia occultae divum perquirere mentis), sembrano riprendere l'analogo scenario dei vv. 47-48 del fr. 6 del De consulatu suo ciceroniano: tum quis non, artis scripta ac monumenta volutans, / voces tristificas chartis promebat Etruscis? (ma vd. già v. 34 Lydius ediderat Tyrrhenae gentis haruspex). Si noti che il quadro ciceroniano richiamato polemicamente da Lucrezio è un quadro stoicheggiante, nel quale le profezie sono segno di un cosmo creato e governato dalla provvidenza divina (cfr. vv. 1-10 principio aetherio flammatus Iuppiter igni /vertitur et totum collustrat lumine mundum / menteque divina caelum terrasque petessit [...] et, si stellarum motus cursusque vagantis / nosse velis [...] omnia iam cernes divina mente notata). Cfr. Volk 2013, 98-99 a proposito delle riprese lucreziane del poema ciceroniano. 51 Le due possibilità prospettate presuppongono come veritiera la proposta interpretativa di Bakker. Anche qualora si ritenesse la Meteorologia siriaco-araba come una sorta di abrégé dei

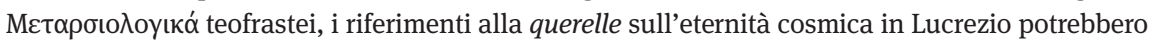
essere egualmente spiegati o come una originale aggiunta del poeta latino o come ripresa di una polemica già presente in Epicuro, volta a sottolineare le contraddizioni insite nella cosmologia peripatetica.

52 Cfr. pp. 180-183. 
sezioni del sesto libro possa risalire a Epicuro. Si noti, innanzi tutto, che numerosi passaggi richiamano polemicamente il fr. 18 Ross del De philosophia, che, con ogni probabilità, era già stato oggetto di confutazione da parte di Epicuro stesso, in quanto prima sintesi della dottrina dell'eternità cosmica. Pare inoltre inverosimile che Epicuro non avesse tratto profitto della propria disamina dei fenomeni naturali più violenti per rilevare le contraddizioni della cosmologia peripatetica, soprattutto in riferimento all'eternità cosmica. ${ }^{53}$ Non si dimentichi infatti che, secondo le ricostruzioni dei contenuti del trattato epicureo, l'esame dei fenomeni naturali avrebbe occupato i libri XII e XIII, subito successivi alla dimostrazione della mortalità del cosmo nel libro XI.

Il ruolo determinante dell'immaginario escatologico nel sesto libro non è però ascrivibile soltanto al modello di Epicuro. Genuinamente lucreziana sembra, ad esempio, l'amplificatio retorica a cui sono soggetti i passi riferiti al tema della fine del mondo. Ad esempio, il crescendo dei versi finali della sezione sui terremoti, culminante nella visione dell'avvento del caos (v. 607), è certo da ascrivere al gusto e alla tecnica poetica di Lucrezio. ${ }^{54}$ Lo stesso vale, probabilmente, per lo scenario catastrofico posto al principio della sezione dedicata al vulcanesimo. Per quanto concerne poi le fonti lucreziane, la presenza pervasiva del $\mu \alpha \kappa \rho \alpha ́ v \theta \rho \omega \pi о \varsigma$, reinterpretato allo scopo di suggerire che il corpo del mondo è mortale come il corpo di un essere umano, così come la presenza di formule riconducibili al dibattito contemporaneo (e.g. aeterna mundi salus), inducono a supporre che Lucrezio abbia utilizzato anche fonti peripatetiche recenziori, forse attinte a testi dossografici. ${ }^{55}$ Come si è visto, questo è il quadro che è emerso anche dall'analisi della sezione escatologica finale del secondo libro, in cui la “traccia” fornita dalla dottrina epicurea dello sviluppo del mondo è integrata con l'uso insistito di modelli analogici volti a confutare anche le più recenti difese peripatetiche dell'eternità cosmica, probabilmente riprese da fonti dossografiche.

53 Contro quale modello peripatetico aveva dunque argomentato Epicuro nella sezione meteorologica del trattato Sulla natura? Si noti che, accettando la proposta di Bakker a proposito della Meteorologia siriaco-araba, l'opzione più probabile è rappresentata dal De philosophia di

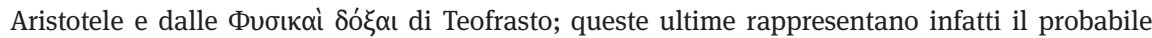
obbiettivo polemico dei vv. 235-379 del quinto libro lucreziano. Per quanto invece riguarda i

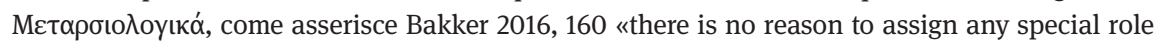
to Theophrastus' meteorological treatise in the formation of Epicurean meteorology».

54 Cfr. a questo proposito Galzerano 2017. Come si vedrà nelle conclusioni relative al sublime lucreziano, questa tendenza non è riconducibile a sole ragioni estetiche; essa sembra invece rispondere anche a un preciso intento didattico e morale.

55 Cfr. Bakker 2016, 157. 
Il confronto di Lucrezio con una tradizione letteraria e filosofica più recente trova ulteriori conferme quando si rivolge l'attenzione alla seconda metà del sesto libro del De rerum natura, che tratta, come già si è accennato, alcuni fenomeni naturali irregolari e locali, considerati fonte di stupore ( $\theta \alpha u ́ \mu \alpha \tau \alpha)$. Per questa seconda parte non si riscontra un preciso parallelo nei testi di Epicuro; è quindi possibile, come supposto da Bakker, che qui il poeta si riferisca alla letteratura paradossografica. ${ }^{56} \mathrm{Si}$ noti che anche Porter evidenzia che la sequenza di argomenti affrontati da Lucrezio in seguito ai terremoti (ovverosia vulcani, acque marine, il Nilo) trova un analogo soltanto nel trattato Del sublime (35.3-5), facendo così pensare a una comune fonte paradossografica, diversamente rielaborata dai due autori. ${ }^{57}$

In questa nuova sezione la tematica della fine del mondo non appare più in forma esplicita: solamente a un livello simbolico l'escatologia cosmica può essere chiamata in causa in relazione al finale del libro, come si vedrà. Lo scopo del poeta appare quello di estirpare lo stupore dalla contemplazione dei fenomeni naturali, per evitare che l'uomo ricada nell'errore fatale di ascrivere all'azione della divinità ciò che gli appare inspiegabile e straordinario. Del resto, in un passo dal De providentia (2.90) che verrà analizzato nel prossimo capitolo, Filone Alessandrino ci mostra come il richiamo a fenomeni naturali irregolari e dannosi per l'uomo fosse impiegato da parte degli Epicurei e degli Scettici contro

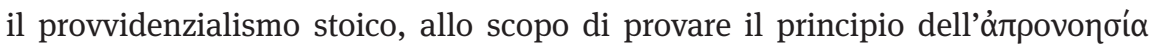
("assenza di provvidenza"). ${ }^{58}$ Non pare un caso che eventi naturali devastanti simili a quelli esaminati da Lucrezio siano richiamati anche nel De providentia di

56 Cfr. Bakker 2016, 109-110; 126-127. Un esempio di trattatatistica paradossografica è il testo pseudo-aristotelico De mirabilibus auscultationibus: si vedano ad esempio i capp. 38 e 40, dedicati alle eruzioni vulcaniche dell'Etna. Nel testo è inoltre incluso l'edificante racconto dei pii fratres risparmiati dall'eruzione dell'Etna, presente anche nel De mundo (400a33-400b6), a proposito del quale cfr. Santelia 2012. È tuttavia necessaria cautela nell'indagine di tali fonti, poiché molto di questo materiale è probabilmente a sua volta attinto da Teofrasto: cfr. Vanotti 1997, 94-96.

57 Cfr. Porter 2007, 172: «if Longinus is indeed quoting from some tradition of paradoxography, Lucretius might seem to be relativising it».

58 Non si può inoltre escludere che Lucrezio, riprendendo il genere paradossografico, si sia confrontato anche con la riflessione della Stoà. Si veda ad esempio Strabone (geogr. 1.2.8; 1.3.16), che dimostra come vi fosse anche una tradizione stoica volta ad analizzare i fenomeni naturali, (anche quelli più violenti, come terremoti ed eruzioni) allo scopo di estirpare lo stupore e la paura dinanzi alla natura. Lo scopo di tale analisi è però opposto a quello lucreziano, poiché essa è tesa a mettere in rilievo il governo provvidenziale del cosmo. Si noti che l'aspirazione alla $\dot{\alpha} \theta \alpha u \mu \alpha \sigma \tau i ́ \alpha$ dinanzi alla natura da parte del saggio è un motivo riconducibile a Democrito; cfr. Barnes 2005, 422. 
Seneca (capp. 2-3), ${ }^{59}$ nel corso di una polemica anti-epicurea, allo scopo di evidenziare come ogni evento naturale sia riconducibile a un progetto divino. Questi raffronti permettono di comprendere come l'accettazione della prospettiva di una ripresa di Epicuro nella prima metà del libro non precluda il riconoscimento dell'accesso, da parte di Lucrezio, a una tradizione filosofica più recente.

\subsection{Le molteplici cause della fine del mondo}

Il tema dell'ignoranza delle cause svolge un ruolo fondamentale nella rassegna

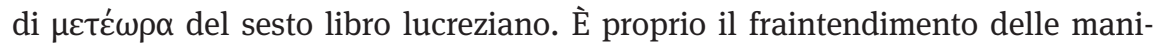
festazioni della forza della natura, imputate a imperscrutabili entità sovrannaturali, oppure a un'onnipotente natura ostile all'umanità, a far cadere l'umanità nell'angoscia e nell'infelicità, rendendola schiava della superstizione. Come si è visto, infatti, Lucrezio è solito impostare la propria analisi di un fenomeno naturale mediante il richiamo all'impressione che esso suscita negli animi (essenziale il ricorso al verbo videri) per poi introdurre la vera ratio, ovverosia la sua spiegazione "scientifica". ${ }^{60}$ Quest'ultima non costituisce però il fine ultimo del poeta, bensì un mezzo volto a raggiungere uno scopo etico: il conseguimento dell' $\alpha \tau \alpha \rho \xi^{\prime} \alpha$ da parte dell'uomo, ormai liberato dalla tirannia della religio. ${ }^{61}$

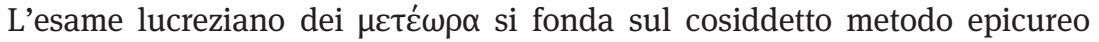
delle spiegazioni multiple. ${ }^{62}$ Nei versi che seguono è il poeta stesso a sintetizzare la propria interpretazione di tale metodo (vv. 703-711): ${ }^{63}$

59 Sen. prov. 2-3 ne illa quidem quae videntur confusa et incerta, pluvias dico nubesque et elisorum fulminum iactus et incendia ruptis montium verticibus effusa, tremores labantis pars rerum circa terras movet, sine ratione, quamvis subita sint, soli aliaque quae tumultuosa accidunt, sed suas et illa causas habent non minus quam quae alienis locis conspecta miraculo sunt, ut in mediis fluctibus calentes aquae et nova insularum in vasto exilientium mari spatia.

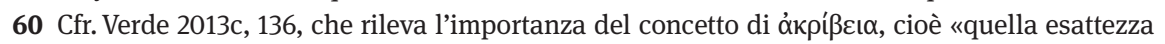
legata non a conoscenze scientifiche fini a se stesse, ma, se confermate e non smentite dai fenomeni, connesse all'ottenimento della felicità».

61 Cfr. Verde 2013c, 141: «il frequente ricorso lucreziano all'analogia con "realtà famigliari” e direttamente percepibili non può che essere il segno chiaro dell'intento terapeutico e perfino di 'rassicurazione' a cui Lucrezio mira». A questo proposito cfr. Clay 1983, 205-212, Hardie 2009b, 231-263 ed Erler 2013, 141.

62 Cfr. Verde 2013c, 139: «è propriamente al cap. 80 della Lettera a Erodoto che Epicuro spiega il metodo delle molteplici spiegazioni in riferimento a $\tau \dot{\alpha} \mu \varepsilon \tau \varepsilon ́ \omega \rho \alpha$. Il punto di partenza è la considerazione empirica e diretta [...] dei diversi modi in cui si genera [...] ciò che è simile [...] al fenomeno celeste di cui s'intendono investigare le cause. Il fenomeno celeste appartiene per- 
Sunt aliquot quoque res quarum unam dicere causam non satis est, verum pluris, unde una tamen sit; corpus ut exanimum siquod procul ipse iacere 705 conspicias hominis, fit ut omnis dicere causas conveniat leti, dicatur ut illius una.

Nam neque eum ferro nec frigore vincere possis interiisse neque a morbo neque forte veneno, verum aliquid genere esse ex hoc quod contigit ei scimus. Item in multis hoc rebus dicere habemus.

703 aliquot $O^{1}$ : aliquod $O Q \quad 705$ iacere $I 31$ : iaceret $O Q \quad 708$ neque : $/ 31:$ que $O Q \quad 710$ verum Marullus: utrum $O Q$ aliquid genere $l 31$ : genere aliquid $O Q$ contigit ei : Vossius : contioitel $O$ : contioite $Q$

Vi sono anche eventi dei quali non basta enunciare una sola causa: ne occorrono molte fra cui sia l'unica vera. Come, se vedi giacere lontano 705

il corpo esanime d'un uomo, conviene enumerare tutte le cause di morte, affinché si giunga a dire quella specifica.

Infatti non puoi intuire dapprima se costui sia perito di spada o di freddo, o di malattia, oppure per caso di veleno, ma sappiamo che è stato qualcosa di tal genere a colpirlo. 710 Ugualmente dobbiamo dire questo in molte circostanze.

Alcuni fenomeni naturali, seppur dovuti a una sola causa, devono essere motivati ricorrendo a più cause (v. 704 pluris): l'impossibilità di una visione chiara e "ravvicinata" di questi eventi (v. 705 procul) e di una comprensione globale delle loro dinamiche impedisce infatti agli uomini di proporre una sola spiegazione valida. Per illustrare tale concetto Lucrezio ricorre poi al noto esempio del cadavere visto da lontano (vv. 705-711): dato che il corpo non è osservabile da vicino, la causa della morte non è determinabile con certezza e, di conseguenza, appare necessario proporre tutte le possibili cause, sapendo che quella vera rientra necessariamente in tale elenco (v. 708-709).

Lo stesso principio è valido anche su scala macrocosmica, se compiano l'esperimento mentale d'immaginare di osservare il corpus exanimum del mondo dopo la sua distruzione. Come si è visto, è il poeta stesso induce il lettore a questa associazione, collocando i versi appena citati subito dopo la disamina dei

tanto, in ultima analisi, agli $\alpha \delta \eta \lambda \alpha$, le cose che, non essendo direttamente oggetti di percezione, sono non evidenti». Cfr. 132 n. 5 a proposito della questione delle possibili fonti peripatetiche di questo metodo.

63 Cfr. il commento di Verde 2013c, 137-141. 
sismi e del vulcanesimo, pervasa dal modello analogico del $\mu \alpha \kappa \rho \alpha ́ v \theta \rho \omega \pi о$ c. $^{64} \mathrm{~A}$ ben vedere, l'esercizio svolto dal lettore lucreziano, soprattutto nell'ultima diade del poema, consiste proprio nel porsi costantemente ante oculos la morte del mondo, cercando di comprenderne le dinamiche e le cause. A differenza del cadavere della similitudine, però, il corpo morente del mondo non può essere visto ricorrendo ai sensi (se non nel momento stesso della fine), bensì affidandosi al ragionamento (5.108-109 et ratio potius quam res persuadeat ipsa / succidere horrisono posse omnia victa fragore).

Secondo Lucrezio, il mondo è soggetto a svariate possibili cause di distruzione. ${ }^{65}$ Del resto, come evidenziato nel corso dell'esame dei vulcani, il numero immenso di atomi che affluiscono contro le mura del mondo dall'infinito spazio esterno (cfr. 662 sunt multarum semina rerum) può apportare numerosissime tipologie di catastrofe. Lo stesso principio era già stato ribadito ai vv. 485-494, dove il poeta aveva riaffermato l'infinità del cosmo (summam profundi / esse infinitam docui) e l'innumerevole numero di atomi che solcano lo spazio, negando il carattere stupefacente (haud igitur mirumst) dell'avvento repentino di oscure tempeste sul mondo (tempestas atque tenebrae / coperiant maria ac terras impensa superne). ${ }^{66}$ Si può però risalire più indietro, sino al quinto libro, dove questa conturbante abbondanza di possibilità di ruina era stata condensata nell'immagine della ianua leti spalancata sul cosmo (patet), o addirittura pronta a inghiottirlo "come le fauci di un'immane voragine" (immani hiatu). ${ }^{67}$

Come si è detto, per spiegare i possibili scenari di distruzione che si configurano per il mondo, Lucrezio si avvale sovente del modello analogico del

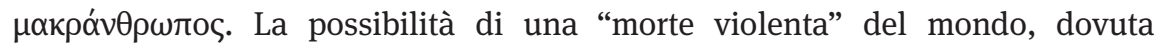
all'improvviso afflusso dall'esterno di atomi apportatori di rovina, viene assimilata alle malattie che possono colpire il corpo umano. Ad esempio, nei già citati vv. 338-350 del quinto libro, i morbi e i pericoli che colpiscono i mortali

64 Cfr. Verde 2013c, 134 che rileva come il ricorso all'analogia divenga dunque «un metodo

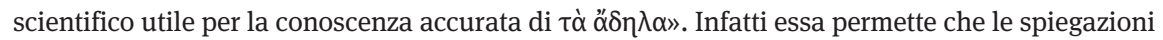
siano sottoposte all' "inaggirabile banco di prova" fornito dalle sensazioni (cfr. Verde 132).

65 La presenza di tali cause di distruzione per i mondi è al contempo la precondizione per la formazione di nuovi mondi. Del resto, come infatti viene sottolineato nel già citato frammento di Metrodoro di Chio (cfr. D.-K. 70 A6), l'esistenza d'innumerabili mondi è resa possibile dalla

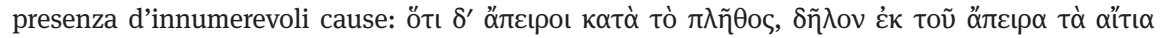

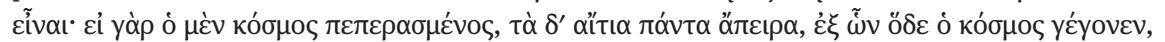

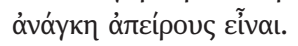

66 Cfr. pp. 337-338.

67 Altro punto di riferimento è il finale del secondo libro (vv. 1105-1149) dove viene descritto l'incessante flusso di atomi dallo spazio infinito, che concorrono alla formazione e alla distruzione dei mondi. 
divengono modello di alcune possibili tipologie di exitium che possono colpire il mondo, come l'incendio cosmico (v. 339), il terremoto (v. 340) o il diluvio (v. 341). Incendio e diluvio vengono richiamati anche nella parte finale della sezione escatologica (vv. 380-415) che evidenzia come un'improvvisa sovrabbondanza di fuoco o di acqua (v. 384 exsuperarint; v. 394 fuerit superantior) potrebbero annientare il mondo. Il terremoto figura invece sia al principio della medesima sezione escatologica (vv. 105-106), nonché nel sesto libro, dove il suo esplicarsi a causa dei venti sotterranei viene comparato ai brividi di freddo che colpiscono un corpo umano (vv. 589-593). Il motivo del $\mu \alpha \kappa \rho \alpha ́ v \theta \rho \omega \pi о \varsigma$ ricorre anche nella prima parte della sezione dedicata ai vulcani nel sesto libro: qui di nuovo le malattie umane sono usate come esempio per dimostrare che anche il mondo può essere soggetto a incendi, diluvi, terremoti e tempeste. L'aggiunta dello scenario del turbo (v. 668 ac terras rapidus percurrere turbo) sembra suggerire implicitamente che il poeta accetti anche la possibilità di catastrofi dovute a un sovrabbondare di aria. ${ }^{68}$

In sostanza, tra le molteplici possibili cause di distruzione del mondo, il poeta sembra soffermarsi su quelle dovute al sovvertimento dell'equilibrio originario, dovuto al superare (sempre causato dall'afflusso di atomi dall'universo) di uno dei quattro elementi: l'aria (turbini e tempeste), il fuoco (l'incendio del cosmo), l'acqua (il diluvio), e la terra (il sisma). In nessun punto del poema Lucrezio esclude che queste cause possano essere compresenti e divenire quindi concause della ruina finale. Ad esempio, molti dei quadri di tempesta descritti nel sesto libro contemplano sia la furia dei venti sia l'avvento del diluvio. Non si deve infine dimenticare che, nel finale del secondo libro, il poeta prospetta l'eventualità che il mondo sia distrutto in un grande crollo, dovuto però non a un eccesso repentino di uno specifico elemento, ma piuttosto a causa della propria vetustas, quale esito del lungo assedio atomico a cui è stato sottoposto (vv. 11441145): sic igitur magni quoque circum moenia mundi / expugnata dabunt labem putrisque ruinas.

Pertanto, servendosi dei modelli analogici sopra evidenziati, Lucrezio mostra che il mondo è soggetto a diverse tipologie di rovina, menzionando sia l'invecchiamento sia eventi improvvisi, come il terremoto, l'incendio, il diluvio e la tempesta. ${ }^{69}$ Egli non specifica però mai che queste siano le sole possibilità: il

68 Cfr. anche 6.564-572, dove si sottolinea che i sismi sono dovuti alla furia dei venti sotterranei. Sul ruolo dei venti nel poema lucreziano, cfr. Leone 2015.

69 La possibilità di opporre la ruina per invecchiamento alle altre forme sembra rispecchiare la sintesi ciceroniana del fr. 2 Buescu degli Aratea, nel quale si dice che il mondo, se mortale, sarebbe soggetto a tempestas oppure a vetustas. Tale opposizione risale, come si è visto, al Timeo 
paragone con le malattie ci fa anzi comprendere che il novero fosse ben più ampio e che le modalità menzionate dal poeta siano state selezionate per la loro potenzialità evocativa e perché più utili a confutare le contraddizioni della dottrina dell'eternità del cosmo. Inoltre, a differenza di Diogene di Enoanda, Lucrezio non asserisce mai che una causa è più persuasiva di un'altra. ${ }^{70}$ Sotto questo profilo, egli si mantiene dunque fedele a Epicuro e rispetta il principio

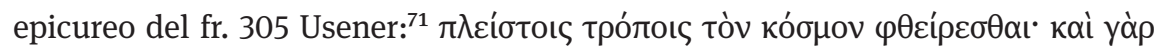

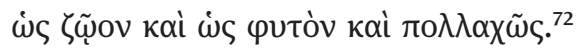

Come evidenziato da Verde, vi sono però alcuni elementi del metodo del

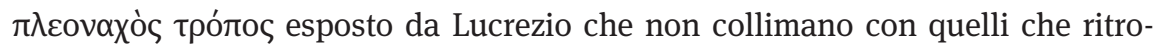
viamo nell'esposizione epicurea dell'Epistola a Pitocle, e che sembrano anzi un suo "superamento". ${ }^{73}$ Ad esempio, citando le cause dei movimenti astrali (5.526-

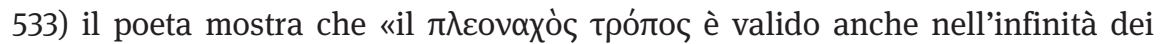
mondi, con la conseguente variabilità dei fenomeni e delle loro cause». ${ }^{74}$ Questa estensione del suo «spettro di applicazione» non è presente nell'Epistola $a \mathrm{Pi}$ -

di Platone e la sua ripresa da parte di Lucrezio potrebbe essere mossa in primis da intenti polemici verso Platone e i suoi eredi, sino alla scuola peripatetica.

70 A proposito della particolare interpretazione delle molteplici cause da parte di Diogene di Enoanda (fr. 13, col. III Smith) e delle sue implicazioni, cfr. Verde 2013c, 135-137. Come si è visto, credo che la particolare enfasi attribuita a diluvi e incendi in 5.380-415 non implichi una predilezione per queste catastrofi, ma sia piuttosto dovuta a motivi di amplificatio retorica in sede conclusiva e, parimenti, all'intento di confutare Platone e Teofrasto, che avevano rivolto attenzione proprio a questo tipo di cataclismi.

71 Come si è visto nell'analisi del finale del secondo libro, Lucrezio sceglie però di esaminare le realtà più familiari al lettore, rivolgendo la propria attenzione ai mondi simili al nostro e alle modalità di distruzione $\dot{\omega} \zeta \zeta \tilde{\omega}$ ov, messe in rilievo tramite il modello analogico del $\mu \alpha \kappa \rho \alpha ́ v-$ $\theta \rho \omega \pi$. . Sono invece lasciate in ombra le questioni della presenza di mondi differenti dal nostro e dell'esistenza di diverse tipologie di ruina.

72 Cfr. Bakker 2016, 21-22. Sottolineando che per gli Epicurei ogni spiegazione è non solo possibile, ma anche necessariamente vera in qualche punto dell'universo infinito, lo studioso enuncia il «principle of plenitude», secondo cui: «although in our world each explanation can at best be called possible, in the universe at large, given the infinity of space and matter and hence of worlds, any given possibility cannot fail to be realised (the "principle of plenitude"), and so every possible explanation is also "true", if not here, then somewhere else».

73 Cfr. Verde 2013c, 139. Diversa è l'opinione di Bakker 2016, 42, secondo il quale, in riferimento alle spiegazioni multiple, il solo Diogene di Enoanda si discosta da Epicuro, mentre Lucrezio resta Fedele al maestro.

74 Lucr. 5.526-533 nam quid in hoc mundo sit eorum ponere certum / difficile est; sed quid possit fiatque per omne / in variis mundis varia per omne; / e quibus una tamen sit et hic quoque causa necessest / quae vegeat motum signis; sed quae ratione creatis, / id doceo plurisque sequor disponere causas, / motibus astrorum quae possint esse sit earum / praecipere haudquaquamst pedetemptim progredientis. 
tocle, nella quale non vi è neppure riferimento «né alla presunta difficoltà, sostenuta, invece, da Lucrezio, di comprendere quale sia la causa davvero operante nella spiegazione di un certo fenomeno celeste, né al fatto che, tra le molte cause avanzate, una sola è propriamente quella davvero responsabile del verificarsi del fenomeno in questione». ${ }^{75}$

Non è però purtroppo chiaro se tali differenze siano dovute a un'evoluzione delle posizioni nella scuola epicurea, ${ }^{76}$ a un'interpretazione (o fraintedimento) del maestro da parte di Lucrezio, ${ }^{77}$ o, ancora, al fatto che Epicuro stesso avesse presentato nel trattato Sulla natura una versione "aggiornata” di tale metodo, e che di essa il poeta ci renda conto. ${ }^{78}$ L'esame qui svolto a proposito dell'escatologia cosmica offre probabilmente maggiori conferme alla possibilità che Lucrezio richiami uno sviluppo più recente della dottrina delle cause multiple nella scuola epicurea: tale dato rispecchierebbe in effetti il quadro, emerso in questo lavoro, di un Lucrezio fedele a Epicuro, ma al contempo pronto a richiamare il dibattito filosofico successivo alla morte del maestro allo scopo di attualizzarne il messaggio. In effetti, i pochi dati a disposizione sembrano riscontrare una certa autonomia del poeta: egli, pur accettando l'idea che il mondo vada in rovina in molti modi, non conferisce egual attenzione alle possibilità prospettate

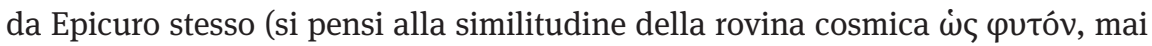
menzionata o sviluppata dal poeta) ma solamente a quelle più utili ai propri intenti (poetici e polemici). ${ }^{79}$

75 Verde 2013c, 139-140.

76 Cfr. Verde 2013c, 142: «dall'analisi fin qui condotta mi sembra lecito desumere che la teoria delle molteplici spiegazioni e la stessa nozione di casualità a essa collegata, dall'Epistola $a$ Pitocle ai frammenti litici di Enoanda, abbiano avuto una significativa 'evoluzione' e abbiano costituito una delle dottrine più frequentate della tradizione epicurea, che non ha risparmiato, pertanto, esegesi e interpretazioni dell'originaria formulazione di Epicuro».

77 Cfr. Masi 2014 60-61, che propone la nozione di ascrivere le cause multiple epicuree alla nozione di "multiple causal possibilities", che non sono né spiegazioni né cause, ma «the multiple potentialities of occurring actually possessed by the bodies affected by meteorological and astronomical processes. These possibilities, moreover, are not necessarily mutually exclusive, as Lucretius and contemporary critics would have it».

78 Cfr. Verde 2013c, 141-142: «non è impossibile che dell'evoluzione di questa specifica dottrina sia responsabile Epicuro stesso nel $P F$, tenendo conto del fatto che i libri XI-XIII di quest'opera erano dedicati a questioni cosmologiche e all'esame dei fenomeni celesti. Se così fosse, il 'fondamentalismo' epicureo di Lucrezio sarebbe confermato».

79 Si consideri ad esempio l'asimmetrica attenzione data al terremoto, all'incendio e al diluvio rispetto alle altre possibili forme di distruzione. 


\subsection{Fine della città e fine del mondo: simboli di escatologia cosmica nella Peste di Atene}

Dopo la trattazione dei fenomeni vulcanici (vv. 639-702), Lucrezio si volge all'analisi di una serie di fenomeni naturali privi delle implicazioni escatologiche che contraddistinguono la prima metà del sesto libro. Alle piene del Nilo (vv. 703-737), seguono i luoghi definiti "Averni” (vv. 738-839), l'escursione termica tra sottosuolo e superficie in pozzi e fontane (vv. 840-905); il magnetismo (vv. 906-1089). L'ultima tipologia di fenomeni analizzati è costituita dalle malattie (vv. 1090-1286): ${ }^{80}$ questa sezione culmina nel celeberrimo episodio esemplare della Peste di Atene ${ }^{81}$ che - com'è noto - è interamente modellato per traccia, struttura e lingua sulla narrazione tucididea nel secondo libro della Guerra del Peloponneso (2.47-53). Nondimeno, quella di Lucrezio non è una mera traduzione, bensì una rielaborazione che assume un valore simbolico universale: ${ }^{82}$ essa può essere interpretata come una sorta di mito epicureo, quasi un contraltare ai grandi miti finali nei dialoghi platonici. ${ }^{83}$

È proprio nella sua valenza mitica e paradigmatica che la narrazione lucreziana dell'epidemia entra in relazione con l'escatologia cosmica. Innanzi tutto, l'episodio della peste può essere letto come un grande exemplum del concetto epicureo di ámpovonó́a (assenza di provvidenza divina). Per la comprensione di quest'ultimo concetto - e, più in generale, del significato del finale

80 Per quanto concerne il ruolo marginale delle malattie nella tradizione meteorologica antica, cfr. Bakker 2016, 124-126.

81 Un punto di rifemento imprescindibile resta l'interpretazione di Commager 1957; per un'introduzione cfr. anche Büchner 1957, 64, Bonelli 1984, 265-290, Labate 1991, Segal 1998, 5260, 28, Schiesaro 2007, 55-58 e Morrison 2013, 219-222. Segnalo inoltre la prossima uscita di un commento di Schiesaro a questo passo e alle sue implicazioni simboliche.

82 Cfr. Dionigi 1990, 610-613: «a parte questa sostanziale convergenza e fedeltà, la rievocazione di Lucrezio è punteggiata di omissioni, aggiunte e alterazioni, che vanno attribuite non già a fraintendimenti (Munro, Giussani, Bailey) o a filtri del modello tucidideo (Ernout), bensì alla trasposizione dal piano oggettivo e descrittivo - proprio dello storico greco, più sensibile ai particolari storici, geografici, fisici e clinici, e condizionato dalla propria esperienza - al piano emotivo e psicologico, ricco di suggestioni morali e universali, prioritarie per Lucrezio». Cfr. anche Segal 1998, 54-55.

83 Cfr. Gale 1994, 225: «like the opening invocation, the plague has also a number of symbolic functions, and several layers of meaning. It can be fruitfully compared to a Platonic myth, presenting moral or psychological truths in a symbolic form with strong visual and emotional impact. One thinks particularly of the Myth of Er at the end of the Republic. Lucretius' myth [...] is thus transformed from a past event into a vehicle for meanings of universal and paradigmatic application». Così interpretato, l'episodio presenta notevoli tangenze con il concetto di "révélation finale", introdotto da Lévi 2014. 
lucreziano - è necessario chiamare in causa un passo dal De providentia di Filone Alessandrino (2.90); l'interlocutore di Filone (Alessandro) ${ }^{84}$ elenca una serie di argomentazioni anti-stoiche volte a provare l'inesistenza della provvidenza tramite il richiamo a fenomeni naturali violenti e dannosi per l'umanità. Questi ultimi coincidono in buona misura con quelli presenti nel sesto libro lucreziano: si citano infatti i terremoti (subitanei motus terrae) poi le pestilenze (pestilentiae), ma, sul fondo, anche il magnetismo (a lapide Herculiano ferro) in relazione alle malattie. Inoltre, nel passaggio filoniano si evidenzia la portata “apocalittica” di tali fenomeni, con motivi topici corrispondenti a quelli presenti nelle sezioni escatologiche del De rerum natura. ${ }^{85}$

L'analogia più notevole tra Filone e Lucrezio consiste però nel fatto che entrambi gli autori chiamano in causa in sede conclusiva, quale exemplum di pestilenza catastrofica, l'episodio della Peste di Atene nella sua narrazione tucididea (nota sub bello Peloponnesiaco pestis fuit, quae a Thucydide refertur). Come Lucrezio, Filone evidenzia non solo le conseguenze fisiche, ma anche le conseguenze psicologiche del fenomeno, che conduce gli uomini alla disperazione (animae amarissimos dolores desperationesque), mettendo in rilievo la loro impotenza. Queste corrispondenze sembrano suggerire una comunanza di fonti tra Lucrezio e Filone: i due autori chiamano in causa il medesimo repertorio di fenomeni naturali per dimostrare l'assenza di provvidenza; come si è visto, tale repertorio fu al centro del dibattito che, in età tardo-ellenistica, vede contrapposti da un lato gli Stoici e, dall'altro, gli Epicurei e l'Accademia scettica. ${ }^{86}$ Ancora una volta, l'impressione è che Lucrezio non si limiti a seguire la traccia di Epicuro, ma la integri con fonti recenziori. ${ }^{87}$ L'enfasi di Lucrezio sul carattere catastrofico e apocalittico dei fenomeni naturali è volta sempre anche a sottolineare la non-provvidenzialità dell'universo, con evidente polemica anti-stoica. ${ }^{88}$ L'originalità lucreziana consiste pertanto non nella scelta dell'episodio in sé e per sé, bensì nella sua elevazione a "grande mito" finale. ${ }^{89}$

84 Per il personaggio di Alessandro nel dialogo filoniano cfr. Hadas-Lebel 1973, 66.

85 Cfr. e.g. Lucr. una dies in 5.95, parimenti riferito a un catastrofico sisma.

86 Cfr. i paralleli richiamati da Holmes 2013, 156 n. 7 «for the pest argument, see Cic. Luc. 2.120, with Reid (1885: 318); Philo prov. 2.56-65; Plut. fr. 193 (Sandbach) [= Porph. abst. 3.20]. Lactant. de ira Dei 13 suggests the argument was associated with the Academics. De Lacy (1948: 19) attributes it more specifically to Carneades and suggests that the Epicureans later appropriated it; see also Bailey (1947: ad Lucr. 3.1353); Schmidt (1990: 200-201); cfr. Sedley (1998: 74 n. 60)».

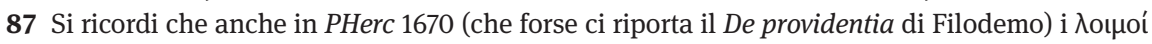
sono menzionati a riprova della non-provvidenzialità del mondo. Cfr. Ferrario 1972, 78-81.

88 Del resto non mancano studi che, conversamente, sottolineano la presenza di elementi antistoici nel grande proemio. Cfr. e. g. Asmis 1982 e Campbell 2014.

89 Per un'interpretazione della peste come allegoria finale della morte cfr. Segal 1998, 58-59. 
Il richiamo alla tradizione stoica è qui fondamentale anche per un'altra ragione; come si è visto, ${ }^{90}$ sempre nel De providentia di Filone, ritroviamo una dimostrazione della mortalità del mondo che si fonda sull'argomento della consunzione delle sue partes (1.17-19):

\begin{abstract}
Cum enim, ut ostendi, partes mundi per destructionem corrumpi videam, cur non maiores quoque et ipsa elementa, caelum, inquam, et terram, destruenda fore concedam, cum iam de partium corruptione omnes consentiant? 18 [...] Agam luculentissime de de terra atque de aere, non minus ipsam aeris naturalem affectionem considerans, quam animadvertens ipsum nunc varias passiones experiri, et rursum in sanitatem redire: unde etiam medicorum iudicio per commutationes eius oriri morbus statuitur; quoniam languescere per hunc dixere corpora in mundo existentia naturali eius participatione. Qui ergo obnoxius est morbo, tempestati ac corruptioni, quidni ipsa quoque vita non demum privetur? [...] Si quis autem aerem putet immortalem esse, ita ut perpetuum istum permansurum dicat, quomodo quaeso in corpore immortali mortales mori soleant? ${ }^{91}$
\end{abstract}

Pur trattando della corruttibilità di tutti i quattro elementi naturali, Filone si sofferma in particolare sulla terra e sull'aria. Similmente, Lucrezio dedica al tema della senescenza della terra l'intero quadro finale del secondo libro (2.1150-1174), ${ }^{92}$ laddove la sezione conclusiva del sesto libro è dedicata proprio alla corruttibilità dell'aria. Un'altra notevole analogia consiste nel fatto che Filone, a riprova della natura mortale di questo elemento, chiama nuovamente in causa le malattie: il fatto che l'aria sia soggetta ad alterazioni, e dunque a cicli che alternano malattia e guarigione, si riverbera infatti sulla società umana che, partecipando dell'aria, è a sua volta esposta a morbi e a corruzione. La peste di Atene descritta da Lucrezio è in fondo un concreto exemplum del principio esposto da Filone: inoltre, anche per il poeta epicureo il semplice dato della corruptio dell'aria - come anche quello della sterilità della terra - diviene im-

90 Cfr. pp. 182-183.

91 «Poiché infatti, come ho mostrato, vedo le parti del mondo soggette a corruzione attraverso la distruzione, perché non dovrei ammettere che anche gli stessi elementi di maggior rilevanza, il cielo e la terra, sono destinati alla distruzione? Tutti, infatti, concordano a proposito della corruzione di ciascuna parte [...]. Tratterò con abbondanza di argomenti della terra e dell'aria, considerando con attenzione la stessa naturale condizione dell'aria, osservando la quale noto che essa stessa subisce vari mutamenti e perturbazioni, per poi tornare alla salute; per questo motivo, anche secondo il giudizio dei medici, le malattie insorgono quando l'aria muta, poiché per essa s'indeboliscono i corpi che sussistono nel mondo e che per natura hanno parte di essa. Chi dunque può essere soggetto alla malattia, alle intemperie e alla corruzione, e tuttavia non essere soggetto alla privazione della propria esistenza? [...] Se qualcuno ritenesse l'aria immortale, affermando che questa resterà eternamente, in che modo - chiedo - corpi mortali sono soliti morire all'interno di un corpo immortale?».

92 Cfr. Appendice 1. 
plicitamente simbolo e prova della natura mortale del mondo intero. Il quadro catastrofico di questo finale conduce infatti il lettore a porsi la domanda con la quale Filone chiude il passo sopra citato: come può essere definito immortale un corpo che accoglie in sé la morte di tanti esseri viventi?

Quanto detto permette di comprendere un altro aspetto del grande finale del De rerum natura. L'episodio della peste di Atene - grande scenario di distruzione civica - diviene simbolo e sintesi di tutti gli scenari apocalittici presenti nei finali lucreziani, dalla fine del mondo del primo e del secondo libro al trionfo della mors immortalis, la morte di ogni individuo, nella conclusione del terzo. ${ }^{93} \mathrm{Il}$ dissolvimento della civitas rappresenta il perfetto punto d'incontro tra la scala "microcosmica" del dissolvimento individuale e la dimensione "macrocosmica" del dissolvimento del mundus. Del resto, nella prospettiva epicurea, l'uomo, la città e il mondo sono tutti esempi di aggregati atomici che hanno una relazione di continuità: la differenza tra essi è solamente quantitativa, ma non qualitativa. La dinamica della morbida vis che irrompe nella città è dunque la stessa che causa la malattia di ogni individuo e che provoca la disgregazione del mondo. Essa si genera o dall'interno, per putrescenza, (v. 1101-1103) oppure dallo spazio esterno all'aggregato (v. 1099 extrinsecus), per l'incombere di una vis esterna: la ruina di Atene corrisponde a quest'ultimo caso. Come nel finale del secondo libro i moenia mundi crollano per il progressivo l'invecchiamento (v. 1173 paulatim tabescere) e per l'assedio degli atomi dall'immensità circostante (vv. 11441145 expugnata dabunt labem putrisque ruinas), così Atene viene colpita dall'incombere dalla malattia a partire dalle regioni esterne all'Attica (vv. 11401142 penitus veniens Aegypti finibus ortus / aera permensus multum camposque natantis / incubuit tandem populo Pandionis omni) tramite un lento processo di accumulazione di miasmi (cfr. v. 1121 ut nebula ac nubes paulatim repit). ${ }^{94}$

È Lucrezio stesso a "preparare" questa associazione tra la città e il mondo tramite il ricorso, soprattutto nella prima metà del sesto libro, al modello analogico del $\mu \alpha \kappa \rho \alpha ́ v \theta \rho \omega \pi о \varsigma:{ }^{95}$ in molti dei contesti di occorrenza di esso, infatti, Lucrezio spiega i fenomeni violenti che colpiscono il cosmo impiegando come termine di paragone proprio le malattie che colpiscono il corpo umano. Come si è detto, tale modalità esplicativa non è neutra, bensì volta implicitamente a

93 Cfr. Segal 1998, 117-118 «il potere dei processi di disintegrazione - che Lucrezio chiama in breve letum, “destino di morte" - d'inghiottire l'intero universo, ha il suo corrispondente umano nella peste che inghiotte l'intera città di Atene alla fine del poema. Questo è l'equivalente umano, sociale, del caos alla fine del mondo».

94 Cfr. Segal 1998, 128-132 a proposito della rappresentazione della peste come una sorta d'invasione.

95 Cfr. pp. 235-236. 
confermare che il mondo, essendo vulnerabile, non è eterno, bensì mortale. Nel cammino di preparazione al grande finale del libro, il passo più rilevante è senz'altro 6.655-664: qui infatti il poeta illustra la fragilità del mondo a partire dall'exemplum del corpo umano, parimenti soggetto a innumerevoli malattie, scrupolosamente enumerate. La tecnica dell'elenco, la presenza del lessico della medicina, l'attenzione rivolta all'intera sfera anatomica del corpo umano e, al contempo, alla dimensione del dolor sono tutti elementi che prefigurano lo stile e lo spirito della narrazione lucreziana della peste, che quasi già si tocca nel bellissimo verso 664 (unde queat vis immensi procrescere morbi).

Il riconoscimento del carattere simbolico dell'episodio permette di comprendere che Atene è probabilmente intesa da Lucrezio come emblematico

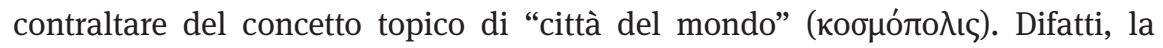
rappresentazione del mondo come ordinata città governata dalla provvidenza divina è un motivo ricorrente nella tradizione filosofica ellenistica, comune ai difensori della teologia astrale, dagli Stoici ai Peripatetici. Per quanto concerne gli Stoici e la cosmopoli, basti considerare numerosi passi dal De natura deorum di Cicerone (cfr. e.g. 2.73-75) ${ }^{96}$; per quanto concerne la tradizione peripatetica a proposito della cosmopoli, è sufficiente volgersi alla trattato De mundo. In quest'ultimo (400b10-30), il mondo è esplicitamente comparato a una città, caratterizzata da un perfetto equilibrio e da un ordine inalterabile; come dice l'autore in un paragrafo precedente (397b2-b8): «le nascite compensano le morti e le morti fanno spazio alle nuove nascite». ${ }^{97}$ Questo ordine non è soltanto prova della provvidenzialità dell'ordine cosmico, ma anche della sua eternità, poiché l'equilibrio degli elementi consente al mondo di restare immutabile nei secoli.

L'Atene di Lucrezio non è certo un esempio di cosmopoli, bensì una vera e propria "città del caos", 98 perfetta antitesi del quadro di vita civile delineato dall'autore del De mundo. Se nel cosmo peripatetico vi è un perfetto equilibrio tra impulsi aggregativi e impulsi disgregativi, ${ }^{99}$ nell'Atene lucreziana vi è un "trionfo della morte" che costituisce il contraltare del "trionfo della vita" col-

96 Si vedano però anche i fr. 20-21 Smith e la confutazione epicurea, da parte di Diogene di Enoanda, della dottrina stoica del mondo come abitazione o città comune agli dèi e al genere umano, discusso alle pp. 145-146.

97 Cfr. Appendice 4.

98 Si noti che l'Atene di Lucrezio può anche essere intesa, in senso empedocleo, come "città della discordia”, soprattutto in relazione agli ultimi versi del poema. Questo aspetto verrà discusso nell'Appendice 2.

99 Si noti che Lucrezio, sulle orme di Epicuro, riprende il medesimo principio di equilibrio isonomico, applicandolo però all'universo e non al mondo: cfr. quanto detto a proposito dei motus exitiales e dei motus auctifici in 2.569-580. 
locato al principio del De rerum natura: ${ }^{100}$ il prepotere dei motus exitiales finisce così per coinvolgere l'intera cittadinanza, senza bilanciamenti o eccezioni (vv. 1285-1286): nec poterat quisquam reperiri, quem neque morbus / nec mors nec luctus temptaret tempore tali. ${ }^{101}$ In questo modo, la narrazione dell'avvento della distruzione e del caos in un preciso periodo storico può essere intesa anche come simbolo universale della condizione e della sorte del nostro mondo e di ogni mondo nell'universo. Il senso profondo dell'espressione tempore tali trascende il remoto contesto storico della guerra del Peloponneso per abbracciare l'hic et nunc di ogni essere umano e del mondo, con riferimento privilegiato alla travagliata contemporaneità del poeta (il patriai tempore iniquo di 1.41). Del resto, come Lucrezio afferma altrove (3.945), eadem sunt omnia semper.

100 Per una sintesi delle interpretazioni del passo cfr. Piergiacomi 2017, 189-201. Per la contrapposizione deliberata tra proemio e conclusione, cfr. Gale 2001a, 32-42.

101 Sono propenso ad accettare la trasposizione di Bockemüller dei versi 1247-1251 in sede conclusiva del libro e dell'opera. Come sottolinea Schiesaro 1994, 92: «death and mourning mors and luctus - adorn the very last line of the poem, with disease (morbus) in the last foot of the penultimate». Una persuasiva serie di argomenti e paralleli testuali a conferma della trasposizione sono addotti da Fowler 1997 (ma cfr. Deufert 2018, 479-483). 